\title{
Mining At Most Top-K\% Spatiotemporal Co-occurrence Patterns in Datasets with Extended Spatial Representations
}

KARTHIK GANESAN PILLAI, Montana State University

RAFAL A. ANGRYK, Georgia State University

JUAN M. BANDA, Stanford University

DUSTIN KEMPTON, BERKAY AYDIN, and PETRUS C. MARTENS, Georgia State University

Spatiotemporal co-occurrence patterns (STCOPs) in datasets with extended spatial representations are two or more different event types, represented as polygons evolving in time, whose instances often occur together in both space and time. Finding STCOPs is an important problem in domains such as weather monitoring, wildlife migration, and solar physics. Nevertheless, in real life, it is difficult to find a suitable prevalence threshold without prior domain-specific knowledge. In this article, we focus our work on the problem of mining at most top-K\% of STCOPs from continuously evolving spatiotemporal events that have polygon-like representations, without using a user-specified prevalence threshold.

CCS Concepts: $\bullet$ Computing methodologies $\rightarrow$ Unsupervised learning;

Additional Key Words and Phrases: Evolving spatiotemporal events, extended spatial representations, spatiotemporal co-occurrence patterns

ACM Reference Format:

Karthik Ganesan Pillai, Rafal A. Angryk, Juan M. Banda, Dustin Kempton, Berkay Aydin, and Petrus C. Martens. 2016. Mining at most top-k\% spatiotemporal co-occurrence patterns in datasets with extended spatial representations. ACM Trans. Spatial Algorithms Syst. 2, 3, Article 10 (September 2016), 27 pages.

DOI: http://dx.doi.org/10.1145/2936775

\section{INTRODUCTION}

Given a spatiotemporal database in which the data objects continuously change their movement, shape, and size, our aim is to discover at most top-K\% of STCOPs (TopK\%STCOPs) that represent two or more different event types, whose instances often occur together in space and time. Top-K\%STCOPs represent a pattern set whose prevalence measures is greater than zero, and the patterns are in the top-K\% of the complete set of STCOPs.

Identifying Top-K\%STCOPs in datasets with evolving extended (i.e., region-based) spatial representations is an important problem for many application domains,

This work was supported by two National Aeronautics and Space Administration (NASA) grant awards, No. NNX09AB03G and No. NNX11AM13A, and by National Science Foundation (NSF) grant award No. 1443061.

Authors' addresses: K. Ganesan Pillai, Gianforte School of Computing, 357 Barnard Hall, Montana State University, Bozeman, MT 59717; email: karthikgp@gmail.com; R. A. Angryk, D. Kempton, and B. Aydin, Department of Computer Science, P.O. Box 5060, Georgia State University, Atlanta, GA 30302-5060; emails: \{angryk, dkempton1, baydin2\}@cs.gsu.edu; P. C. Martens, Physics Department, P.O. BOX 173840, Montana State University, Bozeman, MT 59717-3840; email: martens@astro.gsu.edu; J. M. Banda, Center for Biomedical Informatics Research, Room X-233, 1265 Welch Road, Stanford University, Stanford, California 94305; email: jmbanda@stanford.edu.

Permission to make digital or hard copies of part or all of this work for personal or classroom use is granted without fee provided that copies are not made or distributed for profit or commercial advantage and that copies show this notice on the first page or initial screen of a display along with the full citation. Copyrights for components of this work owned by others than ACM must be honored. Abstracting with credit is permitted. To copy otherwise, to republish, to post on servers, to redistribute to lists, or to use any component of this work in other works requires prior specific permission and/or a fee. Permissions may be requested from Publications Dept., ACM, Inc., 2 Penn Plaza, Suite 701, New York, NY 10121-0701 USA, fax +1 (212) 869-0481, or permissions@acm.org.

(c) 2016 ACM 2374-0353/2016/09-ART10 $\$ 15.00$

DOI: http://dx.doi.org/10.1145/2936775 

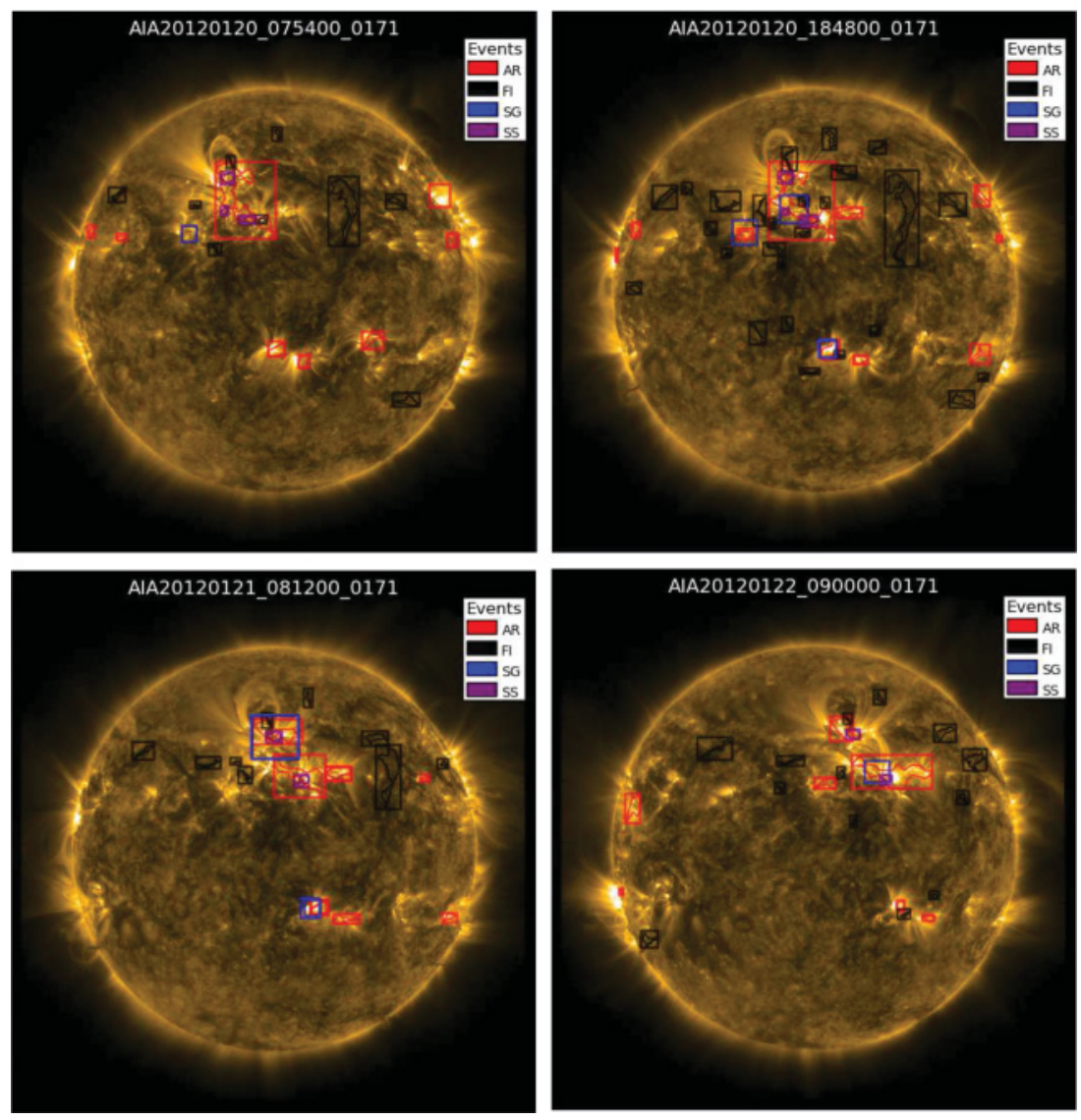

Fig. 1. Images showing the spatiotemporal evolution of four types of solar events (National Aeronautics and Space Administration (NASA) instrument and timestamp are printed on the top of each image).

including weather monitoring, wildlife migration [Lakshmanan et al. 2010], and solar physics [Martens et al. 2012], which is our application focus.

A Real-life Example: Discovering Top-K\%STCOPs is of high importance in solar physics, in which STCOPs frequently occur among various solar events. Figure 1 shows four types of solar events: Active Regions (AR), Filaments (FL), Sigmoids (SG), and Sunspots (SS). As seen in Figure 1, the shapes of the solar events are represented as extended spatial representations (polygons). The shape, size, and location of the solar events continuously evolve over time as shown in the time-series of images in Figure 1. All of these factors influence relationships between various solar events, which lead to complex spatial and temporal interactions. The discovery of the Top-K\%STCOPs only recently became possible for solar data, due to the recently launched National Aeronautics and Space Administration (NASA) Solar Dynamics Observatory (SDO) telescope that registers solar events with spatial and temporal resolution never available before and the decade-long colloboration of Angryk and Martens [Lamb et al. 2008; Martens et al. 2012]. Discovering the STCOPs on the sun is highly important since it could help us to better understand relationships between solar events, and lead to better modeling and forecasting of important solar events that have a significant impact on space weather. Solar events include coronal mass ejections and solar flares, which impact radiation in space, thus can influence space and air travel, intercontinental 
communications (via disturbing satellites), and even cause problems with power grids [Langhof and Straume 2011].

The STCOPs mining algorithms proposed in our initial works [Ganesan Pillai et al. $2012,2013,2014]$ required users to provide an accurate prevalence thresholds value. First, choosing a small prevalence threshold value usually generates many patterns that quickly exhaust our computational resources. A large prevalence threshold value can prune a lot of significantly interesting patterns, or no patterns at all. Moreover, a small set of highly frequent patterns may not be interesting to the user due to the reporting of patterns that are so frequent that they are already well known to the domain experts. Also, the probability of STCOPs of large-size co-occurrence is inherently smaller than that of co-occurrence of smaller -size patterns. Thus, we can argue against using the same prevalence threshold value for STCOPs of all sizes. In classical shopping data analysis, association rule discovery from patterns of frequently purchased items is an important area of growing research. The task of discovering STCOPs is similar to the classic task of discovering items that are frequently bought together from shopping basket data mining. However, it is significantly harder than the frequentitems mining problem because of the lack of transactions and continuous character of spatiotemporal data. In market-basket datasets, transactions represent sets of item types bought together by customers. In the STCOPs mining problem, transactions are not often explicit. Shopping transactions are often disjoint in the sense of not sharing instances of item type. In the spatiotemporal co-occurrence problem, the instances of spatiotemporal events are embedded in a space and time, sharing a variety of spatiotemporal relationships with each other. As in the shopping basket analysis, there is no preassumed thresholds for frequent or nonfrequent patterns. If we set threshold values too high, we get the obvious results (e.g., people buying diapers also buy formula), too low and number of patterns is overwhelming. A similar unsolved challenge is part of our work. Inspired by the work on the spatial co-location problem [Huang et al. 2004], we followed the event-centric model to capture the spatiotemporal Overlap relation between instances of different event types. Specifically, we used a co-occurrence coefficient (cce) to assess the strength of the spatiotemporal Overlap relation between instances of different event types. Moreover, we compared two measures, Jaccard and $O M A X$, that have the useful antimonotonic property (i.e., monotonically nonincreasing when the size of the pattern instance increases), to assess the spatiotemporal overlap relation strength. Next, we used a participation index ( $p i$ ) [Huang et al. 2004] to measure the prevalence of co-occurrence patterns using the count of instances in the event types that satisfy the spatiotemporal relation Overlap. Note that a participation index is also used to rank the spatiotemporal patterns generated to find the percentage of number of top STCOPs to be mined.

It is far from trivial to extend our work presented in Ganesan Pillai et al. [2012] to discover Top-K\%STCOPs. The memory and computational costs involved in extending our original algorithms [Ganesan Pillai et al. 2012, 2013, 2014] to mine Top-K\%STCOPs are significant in comparison to the proposed approach. We will demonstrate these costs clearly in our experimental analysis.

Contributions: This article makes the following new contributions: (1) We define the Top-K\%STCOPs mining problem for extended spatial representations that evolve over time. (2) We propose a novel and computationally efficient FastTop-K\%STCOPs mining algorithm to discover Top-K\%STCOPs and generate spatiotemporal co-occurrence rules from the STCOPs discovered. (3) We compare and report results, and highlight the computational efficiency of our novel FastTop-K\%STCOPs algorithm over the naïve alternative and our original (i.e., not a Top-K\% intended algorithm [Ganesan Pillai et al. 2012, 2013, 2014]). (4) We compare two measures that have the useful antimonotonic property. We do this in order to assess the strength of the spatiotemporal Overlap relation and provide theoretical proof of the selectivity of these measures when they 
are applied to datasets with time-evolving polygon representations. We then discuss important aspects of these measures in spatiotemporal data mining (e.g., storage and computational costs for calculating unions and intersections of trajectories of $2 D$ objects that evolve in time, and experimental validation of our theoretical proofs). (5) We experimentally evaluate the proposed Top-K\%STCOPs mining algorithms using three real-life datasets that contains event types with drastically different spatial and temporal characteristics.

Scope: This article focuses on the Top-K\%STCOPs applied to datasets with extended spatial representations that evolve over time. We use the Top-K\%STCOPs approach to eliminate the previously necessary [Ganesan Pillai et al. 2012, 2013, 2014] userspecified prevalence threshold. The following issues are beyond the scope of this paper: (1) determining the minimal threshold values to capture the strength of spatiotemporal neighborhood relations between instances of different event types; (2) spatiotemporal data-indexing strategies related to speeding up the process of mining STCOPs [Aydin et al. 2014]; and (3) Comparison with non-Apriori approaches [Ganesan Pillai 2014b].

The rest of the article is organized as follows: Section 2 gives a background on related work in which we review important concepts of modeling STCOPs. We also provide theoretical proof on the selectivity of our measures in Section 3. We present basic concepts in order to provide a formal model of Top-K\%STCOPs. We then present the problem statement of mining Top-K\%STCOPs in datasets with evolving extended spatial representations in Section 4. In Section 5, we present a variety of experiments that demonstrate the computational efficiency of our algorithm and the effects of our measures on the number of pattern instances generated, such as execution time, memory usage, and rules generated. In Section 6 , we conclude with a summary of results and our plans for future works.

\section{RELATED WORK}

The problem of mining top $\mathrm{K}$ itemsets was first explored in classical (i.e., nonspatial) association pattern mining literature. The problem of mining $\mathrm{N}$-most interesting itemsets was introduced by Fu et al. [2000]. In this article, the authors propose a setting such that the users indicate the amount of results that are required and do not provide a support threshold. The authors introduced two Apriori-based [Agrawal et al. 1993] algorithms named Itemset-Loop and Itemset-iLoop for mining N-most interesting itemsets. Later, Cheung and $\mathrm{Fu}$ [2004] introduced two new algorithms called LOOPBACK and $B O M O$, to mine $\mathrm{N} k$-itemsets with the highest support for $k$ up to a certain $k_{\max }$ value. In the paper, Cheung and Fu show that both algorithms $L O O P B A C K$ and BOMO outperform the Itemset-Loop [Fu et al. 2000] algorithm.

Hirate et al. [2004] proposed the $T F^{2} P$-growth algorithm for mining Top-K itemsets without any threshold. The $T F^{2} P$-growth algorithm mines patterns in descending order of their support values and sequentially returns frequent patterns to the user. Ngan et al. [2005] used techniques of the COFI-tree for mining the N-most interesting $k$-itemsets without a support threshold. The COFI-tree technique, unlike the $F P$ growth algorithm, avoids a vast amount of recursive calls. Ngan et al. [2005] showed that using the COFI-tree technique performs well, especially with small $k$. The proposed approaches in classical association pattern mining to mine the Top-K patterns cannot be directly applied to our Top-K\%STCOPs because of the complexity of spatial datatypes, spatiotemporal relationships, and their requirement of a transaction database [Shekhar and Chawla 2002].

In spatial and spatiotemporal co-location mining literature, state-of-the-art algorithms have been proposed for mining: (1) Spatial co-location patterns [Shekhar and Huang 2001], (2) N-most prevalent co-location patterns [Yoo and Bow 2009], (3) mixed drove co-occurrence mining [Celik et al. 2006], (4) Top-K\% mixed drove co-occurrence 
patterns [Celik et al. 2007], and (5) spatiotemporal co-occurrence pattern mining in datasets with evolving regions [Ganesan Pillai et al. 2012, 2013, 2014].

Shekhar and Huang introduced the problem of mining spatial co-location patterns in Shekhar and Huang [2001]. Shekhar and Huang [2001] define spatial co-location patterns as a subset of spatial events that are frequently located together. Also, the authors introduced an Apriori-based algorithm called Co-location Miner, to mine spatial co-location patterns. The Co-location Miner algorithm requires users to specify a support threshold. Yoo and Bow [2009] introduced the problem of finding the N-most prevalent spatial co-location patterns. Yoo and Bow [2009] use an FP-growth algorithm for finding $\mathrm{N}$-most prevalent co-location patterns, in which users specify a threshold on the amount of results instead of a specified prevalence threshold. These two methods are limited to datasets with just spatial point representations (not $2 D$, as in our investigation), and do not consider temporal information about the spatial data.

Celik et al. [2006] introduced the problem of mining mixed drove spatiotemporal co-occurrence patterns (MDCOPs). They define MDCOPs as a subset of different spatiotemporal event types whose instances are neighbors in space and time. Celik et al. [2006] introduced an Apriori-based algorithm called MDCOP-Miner. However, the MDCOP-Miner algorithm requires users to specify spatial and temporal prevalence thresholds. Celik et al. [2007] introduced the problem of mining the Top-K\%MDCOPs and extend their earlier work [Celik et al. 2006] to discover the Top-K\%MDCOPs, without using user-specified spatial and temporal prevalence thresholds. In the TopK\%MDCOPs algorithm, the authors use a filtering step in each step of the algorithm, and they maintain a top-K\% candidate list until the correct Top-K\%MDCOPs are selected.

These two approaches [Celik et al. 2006, 2007] are limited to datasets with just spatial-point representations, thus cannot be directly applied on the data with polygonlike representations. Moreover, the algorithm proposed in Celik et al. [2007] uses a spatial prevalence index for each time slot and a temporal prevalence index across all time slots to find a candidate top-K\% list.

This article is a novel approach to discover Top-K\%STCOPs motivated by our recent work in Ganesan Pillai et al. [2012, 2013, 2014], in which we introduced the problem of mining spatiotemporal co-occurrence patterns in datasets with extended spatial representations that evolve over time. In our work, event types are modeled as 3D spatial objects to capture different spatial and temporal characteristics of evolving extended spatial representations. We defined multiple measures to capture the strength of spatiotemporal Overlap between instances of different event types based on volumes resulting from the Overlap (i.e., co-existence of these events) [Ganesan Pillai et al. 2012, 2013, 2014]. Although related, our original works [Ganesan Pillai et al. 2012, 2013, 2014] require a user-specified prevalence threshold, thus, they can not be used to mine the Top-K\%STCOPs without using a prevalence threshold.

\section{BASIC CONCEPTS AND PROBLEM STATEMENT}

\subsection{Modeling STCOPs}

Given a set of spatiotemporal event types $E=\left\{e_{1}, \ldots, e_{M}\right\}$, and a set of instances $I=\left\{i_{1}, \ldots, i_{N}\right\}$ of these event types, which evolve over time such that $M \ll N$, a spatiotemporal co-occurring pattern is a subset of spatiotemporal event types that physically co-occur in both space and time.

In Figure 2, we show an example dataset that we will use to explain the definitions in detail. In Table I, we show the unique Instance ID, Event Type, Start Time, and End Time of each instance in our example dataset of Figure 2. This dataset contains four event types. The event type $e_{1}$ has a total of five spatiotemporal instances $\left(i_{1}-i_{5}\right), e_{2}$ 


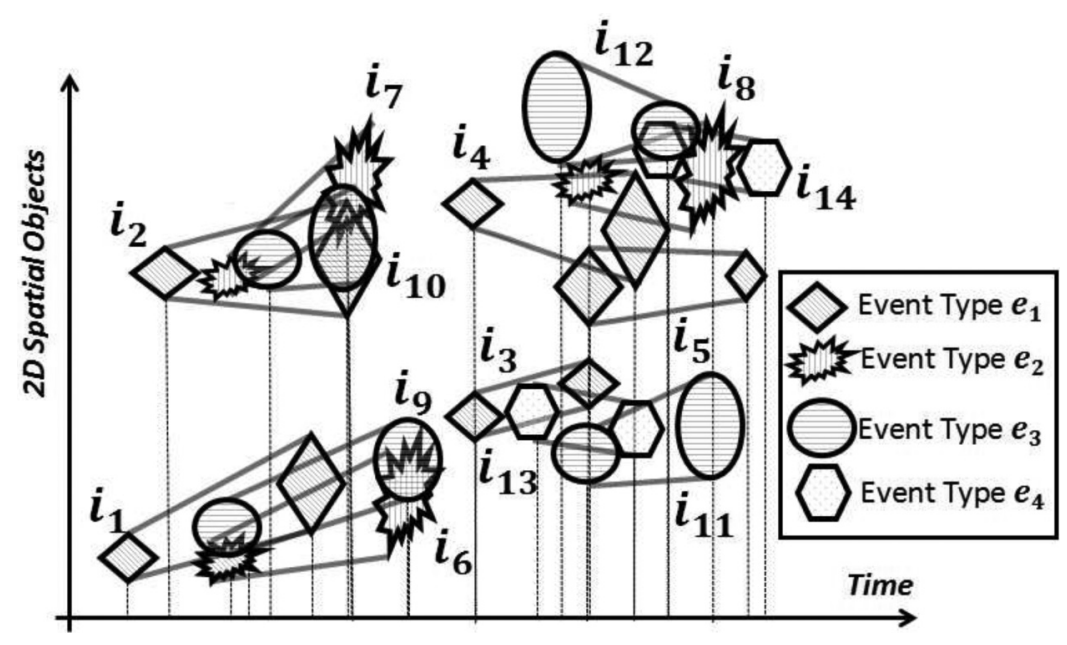

Fig. 2. A spatiotemporal dataset with $2 \mathrm{D}$ spatial objects evolving in time.

Table I. Temporal Information About Event Instances of Data Shown in Figure 2

\begin{tabular}{cccc}
\hline Instance ID & Event Type & Start Time (HH:MM) & End Time (HH:MM) \\
\hline$i_{1}$ & $e_{1}$ & $10: 00$ & $10: 30$ \\
$i_{2}$ & $e_{1}$ & $10: 10$ & $10: 40$ \\
$i_{3}$ & $e_{1}$ & $11: 00$ & $11: 20$ \\
$i_{4}$ & $e_{1}$ & $11: 00$ & $11: 30$ \\
$i_{5}$ & $e_{1}$ & $11: 20$ & $11: 50$ \\
$i_{6}$ & $e_{2}$ & $10: 20$ & $10: 50$ \\
$i_{7}$ & $e_{2}$ & $10: 20$ & $10: 40$ \\
$i_{8}$ & $e_{2}$ & $11: 20$ & $11: 40$ \\
$i_{9}$ & $e_{3}$ & $10: 20$ & $10: 50$ \\
$i_{10}$ & $e_{3}$ & $10: 30$ & $10: 40$ \\
$i_{11}$ & $e_{3}$ & $11: 20$ & $11: 40$ \\
$i_{12}$ & $e_{3}$ & $11: 10$ & $11: 30$ \\
$i_{13}$ & $e_{4}$ & $11: 10$ & $11: 30$ \\
$i_{14}$ & $e_{4}$ & $11: 30$ & $12: 00$ \\
\hline
\end{tabular}

has three instances $\left(i_{6}-i_{8}\right), e_{3}$ has four instances $\left(i_{9}-i_{12}\right)$, and $e_{4}$ has two instances $\left(i_{13}-i_{14}\right)$. For simplicity, in this example we do not show the sequence of $2 D$ shapes that reflect the spatiotemporal evolution of our data. In our example, $M=4$ (since $E=\left\{e_{1}, e_{2}, e_{3}, e_{4}\right\}$ ), and $N=14$ (all instance IDs are listed in the first column of Table I). From our real-life data gathered by the SDO mission [Martens et al. 2012] micro-sample shown in Figure 1, we can derive the dataset similar to the example shown in Figure 2 by following the trajectories of instances of different solar event types. Note that in Figure 1, timestamps are printed on top of each image showing the evolution of instances over time.

Definition 1. A size- $k$ spatiotemporal co-occurrence pattern is denoted as $S E=$ $\left\{e_{1}, \ldots, e_{k}\right\}$, where $S E \subseteq E, S E \neq \emptyset$, and $1<k \leq M$.

We can have multiple size- $k$ spatiotemporal co-occurrence patterns derived from the set $E$. To separate them, we will use subscripts in future definitions (e.g., $S E_{i}$ ) with an arbitrarily chosen subscript to denote uniqueness. For example, $S E_{i} \neq S E_{j}$, where $S E_{i}$ and $S E_{j}$ contain different event types (i.e., at least one event type must be different). 


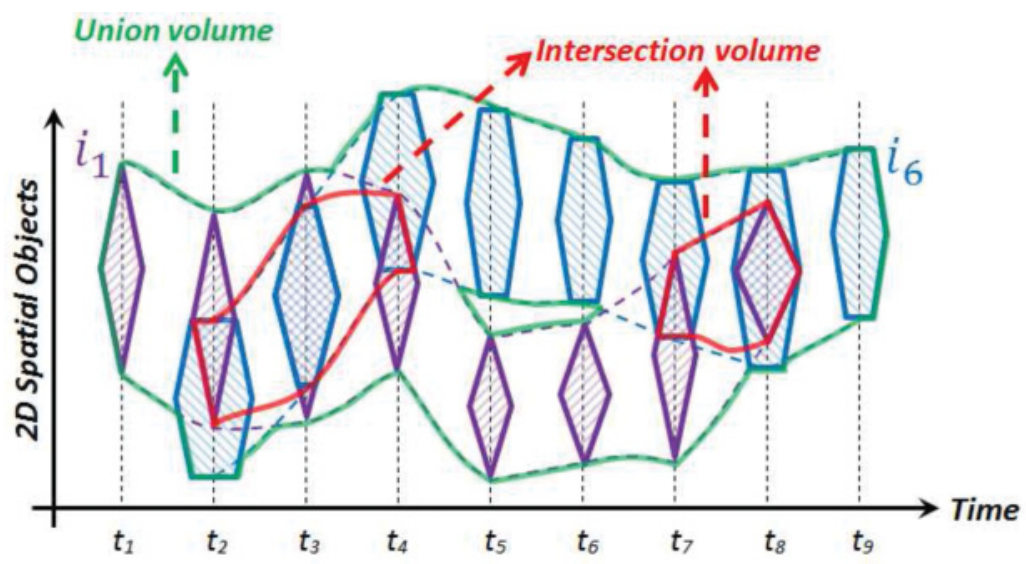

Fig. 3. Example of size-2 co-occurrence of spatiotemporal objects (assume that $i_{1}$ is an instance of spatiotemporal event type $e_{1}$, and $i_{6}$ is an instance of spatiotemporal event type $e_{2}$ ). Red reflects spatiotemporal Intersection volume. Green (in bold) represents spatiotemporal Union volume.

Note that indices $(i$ or $j$ ) do not indicate the number of event types involved in the co-occurrence (we only use $k$ for that).

Definition 2. pat_instance is a pattern instance of a spatiotemporal co-occurrence pattern $S E_{i}$ if and only if pat_instance contains an instance of all event types from $S E_{i}$. No proper subset of patinstance is considered to be a pattern instance of $S E_{i}$.

For example, $\left\{i_{2}, i_{7}, i_{10}\right\}$ is a size-3 $(k=3)$ pattern instance of a co-occurrence $S E_{i}=$ $\left\{e_{1}, e_{2}, e_{3}\right\}$ in the example spatiotemporal dataset (see upper left corner of Figure 2).

Definition 3. A collection of pattern instances of $S E_{i}$ is a table instance of $S E_{i}$, and is denoted as tab_instance $\left(S E_{i}\right)$.

For example, $\left\{\left\{i_{1}, i_{6}, i_{9}\right\},\left\{i_{2}, i_{7}, i_{10}\right\}\right\}$ is a size-3 $(k=3) \operatorname{tab}$ instance $\left(S E_{i}=\left\{e_{1}, e_{2}, e_{3}\right\}\right)$ in the example presented in Figure 2 and Table I.

Definition 4. A prevalent spatiotemporal co-occurrence pattern is of the form $S E_{i}(c c e, p)$, where $S E_{i}$ is a spatiotemporal co-occurrence pattern, and parameters cce, $p$ characterize the prevalent pattern in the following manner:

(1) cce stands for co-occurrence coefficient [Ganesan Pillai et al. 2012]. This is an indicator of the strength of a spatiotemporal relation's occurrence that is investigated. The Naïve STCOPs algorithm uses the spatiotemporal relation Overlap for cce. To distinguish the spatiotemporal relation from the purely spatial one, we will use a capital letter in the name of the former. Some examples of spatiotemporal Overlap are $\left\{i_{1}, i_{6}\right\},\left\{i_{2}, i_{7}\right\}$, and $\left\{i_{7}, i_{10}\right\}$ in Figure 2 . We will discuss this in more detail in Sec. 3.3, and we show an example of spatiotemporal Overlap in Figure 3.

(2) $p$ is the prevalence measure. The prevalence measure emphasizes how interesting the spatiotemporal co-occurrences are, based on their prevalence. In our investigation, we used the participation index ( $p i)$, proposed in Huang et al. [2004], as the prevalence measure. The participation index is antimonotonic when the size of the spatiotemporal co-occurrence pattern increases, which is exploited for computational efficiency similar to Huang et al. [2004].

\subsection{Measures}

Our STCOPs algorithms [Ganesan Pillai et al. 2012] use the spatiotemporal cooccurrence coefficient to calculate cce. The spatiotemporal co-occurrence coefficient 
Table II. Example of a Pattern Instance (Pat_Instance) with Calculation of cce from Data Shown in Figure 3

\begin{tabular}{|c|c|c|c|c|}
\hline $\begin{array}{c}\text { Instance ID } \\
\text { of } e_{1}\end{array}$ & $\begin{array}{c}\text { Instance ID } \\
\text { of } e_{2}\end{array}$ & $\begin{array}{l}\text { Time Instant } \\
(\Delta t=10 \mathrm{~min})\end{array}$ & $\operatorname{Area}\left(i_{1} \cap i_{6}\right)$ & $\operatorname{Area}\left(i_{1} \cup i_{6}\right)$ \\
\hline$i_{1}$ & $i_{6}$ & $t_{1}=10: 00$ & 0 & 60 \\
\hline$i_{1}$ & $i_{6}$ & $t_{2}=10: 10$ & 25 & 120 \\
\hline$i_{1}$ & $i_{6}$ & $t_{3}=10: 20$ & 95 & 115 \\
\hline$i_{1}$ & $i_{6}$ & $t_{4}=10: 30$ & 15 & 140 \\
\hline$i_{1}$ & $i_{6}$ & $t_{5}=10: 40$ & 0 & 150 \\
\hline$i_{1}$ & $i_{6}$ & $t_{6}=10: 50$ & 0 & 140 \\
\hline$i_{1}$ & $i_{6}$ & $t_{7}=11: 00$ & 16 & 130 \\
\hline$i_{1}$ & $i_{6}$ & $t_{8}=11: 10$ & 90 & 90 \\
\hline$i_{1}$ & $i_{6}$ & $t_{9}=11: 20$ & 0 & 60 \\
\hline
\end{tabular}

Note: This pattern instance is a part of Tab_Instance $\left(S E_{i}=\left\{e_{1}, e_{2}\right\}\right)$ as defined in Definition 3, which would include information about all co-occurrences of event types $e_{1}$ and $e_{2}$.

is closely related to the coefficient of areal correspondence (CAC) proposed in Taylor [1977]. CAC is computed for any two (or more, for longer patterns) overlapping polygons as the area of intersection, divided by the area of union (i.e., spatial version of the Jaccard measure). In our works [Ganesan Pillai et al. 2012, 2013, 2014], we extended CAC to three dimensions (two dimensions correspond to space and the third dimension corresponds to time) and calculate the spatiotemporal co-occurrence coefficient using spatiotemporal volumes: (1) the volume of Intersection of the spatiotemporal object's trajectories, and (2) the volume of the Union of their trajectories. We are using volumes because we work with trajectories representing temporal $(1 D)$ evolution of spatial objects with polygon $(2 D)$ representations.

Definition 5. The Intersection volume of a size- $k$ pattern instance, denoted $V\left(i_{1} \cap i_{2} \ldots i_{k-1} \cap i_{k}\right)$, is the volume of the three-dimensional object representing the Intersection of the trajectories of all instances involved in a given pattern instance.

Definition 6. The Union volume of a size- $k$ pattern instance, denoted as $V\left(i_{1} \cup\right.$ $i_{2} \ldots i_{k-1} \cup i_{k}$ ), is the volume of the three-dimensional object representing the Union of the trajectories of all instances involved in a given pattern instance.

\subsection{Co-occurrence Coefficient cce}

We use the spatiotemporal co-occurrence coefficient (cce) as our measure to assess the strength of the spatiotemporal relation Overlap. cce is calculated for a size- $k$ pattern instance as the ratio $J=\frac{V\left(i_{1} \cap i_{2} \ldots i_{k-1} \cap i_{k}\right)}{V\left(i_{1} \cup i_{2} \ldots i_{k-1} \cup i_{k}\right)}$, which is the Jaccard measure [Manning and Schütze 1999] (see Figure 3). We chose the Jaccard measure (J) to capture the spatiotemporal co-occurrence, as it is commonly accepted by data-mining practitioners [Manning and Schütze 1999; Tan et al. 2005; Taylor 1977]. Computing the cce for evolving polygons in very large databases is not a trivial task, both from a computational and storage perspective. In Figure 3, we show the evolution of a pair of instances of two different event types, which change sizes and movement directions across different time instances. We also show the regions of Intersections and Unions at different time slots. The volumes resulting from the Intersection and Union trajectories of objects are shown in Figure 3. If we assume that instances $\left\{i_{1}, i_{6}\right\}$, in our example dataset (Figure 2 and Table I), have spatiotemporal Intersection volume $V\left(i_{1} \cap i_{6}\right)=241$ and a spatiotemporal Union volume $V\left(i_{1} \cup i_{6}\right)=1005$, then the spatiotemporal cce, calculated using Jaccard, is equal to $\frac{V\left(i_{1} \cap i_{6}\right)}{V\left(i_{1} \cup i_{6}\right)}=0.24$. See the notes accompanying Table II for a 
detailed calculation of cce. In Table II, the third column shows time instances (with $\Delta t=10 \mathrm{~min}$ used as our sampling interval), the fourth column Area $\left(i_{1} \cap i_{6}\right)$ shows intersection areas, and the fifth column $\operatorname{Area}\left(i_{1} \cup i_{6}\right)$ shows union areas of these two objects at each time instant. Note that under our current definition for co-occurrence to happen, an explicit intersection must take place between instances of different event types. We can perform spatial operations that use geometric functions to analyze the spatial input data, then generate output data derived from the analysis of input data. In our case, we can use spatial buffer operation ( $S T$ B Buffer) on an input spatial geometry to generate geometry by encircling geometry at a specified distance. The resulting (i.e., buffered) geometries on instances might be large enough to accommodate for instances of different event types that occur close to each other. Although very useful in some real-life applications, discussion on usage of $S T$ Buffer function to discover patterns, whose instances co-occur in neighborhoods instead of physical spatiotemporal join (ST Overlap), is out of the scope of our original interest, since for our work we are interested in co-occurrences that require physical ST Overlap between instances of different event types.

We would like to point out here that computing $J$ for spatiotemporal pattern instances is very expensive (due to the necessary calculations of intersection and union geometries for each timestamp and storage space required to save these geometries). In this article, we will also investigate the alternative measure $O M A X$, defined as $\frac{V\left(i_{1} \cap i_{2} \ldots i_{k-1} \cap i_{k}\right)}{\max \left(V\left(i_{1}\right), \ldots, V\left(i_{k}\right)\right)}$, to assess the spatiotemporal co-occurrence strength (i.e., our cce) of pattern instances.

Egghe and Michel [2002] showed an interesting ordering relation on the selectivity of the measures $J$ and $O M A X$, but their investigation applies only to Boolean values (e.g., items in shopping baskets that can either appear there or not) In this work, we will prove that the ordering relation between $J$ and $O M A X$ also applies to real, positive numbers, and therefore can be utilized to estimate selectivity of these measures when they are applied in our cce calculations.

LEMMA 3.1. The selectivity of the measures $\mathrm{J}$ and OMAX follows the order $J \leq O M A X$, $\forall V \in R^{+}$for $k \geq 2$.

PRoOF. Since numerators are the same for both the measures $J$ and $O M A X$, for the ordering relation $J \leq O M A X$, we can derive relations between both denominators:

$$
\max \left(V\left(i_{1}\right), \ldots, V\left(i_{k}\right)\right) \leq V\left(i_{1} \cup i_{2} \ldots i_{k-1} \cup i_{k}\right) .
$$

One can easily observe that the maximum volume of all trajectories has to be smaller than or equal to the volume of the union of all of them; thus, the relation $J \leq O M A X$ always holds for all positive real numbers.

LEMMA 3.2. The measure $J$ is antimonotone (monotonically nonincreasing), as the size of a pattern instance increases.

PRoOF. The measure $J$ for a size- $k$ pattern instance is defined as

$$
\frac{V\left(i_{1} \cap i_{2} \ldots i_{k-1} \cap i_{k}\right)}{V\left(i_{1} \cup i_{2} \ldots i_{k-1} \cup i_{k}\right)}
$$

For any size- $(k+1)$ pattern instance denoted pat_instance', is equal to pat_instance $U$ $\left(i_{k+1}\right)$, where pat_instance is a size- $k$ pattern instance and $i_{k+1} \notin$ pat_instance. We claim that the measure $J$ follows the relation

$$
\frac{V\left(i_{1} \cap i_{2} \ldots i_{k-1} \cap i_{k}\right)}{V\left(i_{1} \cup i_{2} \ldots i_{k-1} \cup i_{k}\right)} \geq \frac{V\left(i_{1} \cap i_{2} \ldots i_{k-1} \cap i_{k} \cap i_{k+1}\right)}{V\left(i_{1} \cup i_{2} \ldots i_{k-1} \cup i_{k} \cup i_{k+1}\right)} .
$$


Therefore, we need to prove that

$$
V\left(i_{1} \cap i_{2} \ldots i_{k-1} \cap i_{k}\right) \geq V\left(i_{1} \cap i_{2} \ldots i_{k-1} \cap i_{k} \cap i_{k+1}\right),
$$

and

$$
V\left(i_{1} \cup i_{2} \ldots i_{k-1} \cup i_{k}\right) \leq V\left(i_{1} \cup i_{2} \ldots i_{k-1} \cup i_{k} \cup i_{k+1}\right) .
$$

Since adding one more instance of a different event type to a pattern instance can either reduce or not affect the volume of Intersection of instance trajectories, we obtain the relation $V\left(i_{1} \cap i_{2} \ldots i_{k-1} \cap i_{k}\right) \geq V\left(i_{1} \cap i_{2} \ldots i_{k-1} \cap i_{k} \cap i_{k+1}\right)$ in Equation (4). Similarly, adding one more instance of a different event type to the pattern instance can either increase or not affect the volume of Union of instance trajectories; we thus obtain the relation $V\left(i_{1} \cup i_{2} \ldots i_{k-1} \cup i_{k}\right) \leq V\left(i_{1} \cup i_{2} \ldots i_{k-1} \cup i_{k} \cup i_{k+1}\right)$ in Equation (5). Thus, our relation in Equation (3) holds for all positive reals that represent volumes of spatiotemporal objects with evolving polygons.

LEMMA 3.3. The measure OMAX is antimonotone (monotonically nonincreasing) as the size of the pattern instance increases.

Proof. The measure $O M A X$ for a size- $k$ pattern instance is defined as

$$
\frac{V\left(i_{1} \cap i_{2} \ldots i_{k-1} \cap i_{k}\right)}{\max \left(V\left(i_{1}\right), \ldots, V\left(i_{k}\right)\right)}
$$

For any size- $(k+1)$ pattern instance denoted pat_instance' is equal to patinstance $U$ $\left(i_{k+1}\right)$, where pat_instance is a size- $k$ pattern instance and $i_{k+1} \notin$ pat_instance. We claim that the measure $O M A X$ follows the relation

$$
\frac{V\left(i_{1} \cap i_{2} \ldots i_{k-1} \cap i_{k}\right)}{\max \left(V\left(i_{1}\right), \ldots, V\left(i_{k}\right)\right)} \geq \frac{V\left(i_{1} \cap i_{2} \ldots i_{k-1} \cap i_{k} \cap i_{k+1}\right)}{\max \left(V\left(i_{1}\right), \ldots, V\left(i_{k}\right), V\left(i_{k+1}\right)\right)} .
$$

Therefore, we need to prove

$$
V\left(i_{1} \cap i_{2} \ldots i_{k-1} \cap i_{k}\right) \geq V\left(i_{1} \cap i_{2} \ldots i_{k-1} \cap i_{k} \cap i_{k+1}\right)
$$

and

$$
\max \left(V\left(i_{1}\right), \ldots, V\left(i_{k}\right)\right) \leq \max \left(V\left(i_{1}\right), \ldots, V\left(i_{k}\right), V\left(i_{k+1}\right)\right) .
$$

Since, once again, adding one more instance of a different event type to a pattern instance can either reduce or not affect the volume of the Intersection of the instance trajectories, we obtain the relation shown in Equation (8). Similarly, adding another instance of a different event type to a pattern instance cannot reduce the maximum volume of instance trajectories; thus, the relation $\max \left(V\left(i_{1}\right), \ldots, V\left(i_{k}\right)\right) \leq$ $\max \left(V\left(i_{1}\right), \ldots, V\left(i_{k}\right), V\left(i_{k+1}\right)\right)$ holds. Thus, Equation (7) holds for all positive reals that reflect volumes of a spatiotemporal object's evolutions, which are represented as polygons evolving in size and movement direction as time passes by.

\subsection{Prevalence of STCOPs}

Definition 7. The participation index pi of a spatiotemporal co-occurrence $S E_{i}$ is defined as follows:

$$
p i\left(S E_{i}\right)=\min _{j=1}^{k} \operatorname{pr}\left(S E_{i}, e_{j}\right),
$$

where $k$ is the size of the pattern (i.e., cardinality of $S E_{i}$ ), and the participation ratio $\operatorname{pr}\left(S E_{i}, e_{j}\right)$ for a spatiotemporal event type $e_{j}$ is the fraction of the total number of instances of $e_{j}$ forming spatiotemporal co-occurring instances in $S E_{i}$. 
For example, from Figure 2 and Table I, we can see that the pattern instances of $S E_{i}=\left\{e_{1}, e_{2}, e_{3}\right\}$ are $\left\{\left\{i_{1}, i_{6}, i_{9}\right\},\left\{i_{2}, i_{7}, i_{10}\right\}\right\}$. Only two $\left(i_{1}, i_{2}\right)$ out of five instances of the event type $e_{1}$ participate in $S E_{i}=\left\{e_{1}, e_{2}, e_{3}\right\}$. Thus, $\operatorname{pr}\left(\left\{e_{1}, e_{2}, e_{3}\right\}, e_{1}\right)=2 / 5=$ 0.40. Similarly, $\operatorname{pr}\left(\left\{e_{1}, e_{2}, e_{3}\right\}, e_{2}\right)=2 / 3=0.67$, and $\operatorname{pr}\left(\left\{e_{1}, e_{2}, e_{3}\right\}, e_{3}\right)=2 / 4=0.50$. Therefore, the participation index of spatiotemporal co-occurrence $S E_{i}=\left\{e_{1}, e_{2}, e_{3}\right\}$ is $p i\left(\left\{e_{1}, e_{2}, e_{3}\right\}\right)=\min (0.40,0.67,0.50)=0.40$.

Definition 8. The spatiotemporal co-occurrence $S E_{i}$ is a prevalent pattern if it satisfies a user-specified minimum participation index threshold, denoted as $p i_{t h}$.

In our example, if the minimum threshold is set to $p i_{t h}=0.3$, then the spatiotemporal co-occurrence pattern $S E_{i}=\left\{e_{1}, e_{2}, e_{3}\right\}$ is a prevalent pattern.

Nevertheless, the usage of a participation index as a threshold value has drawbacks: (a) Setting a small threshold value can generate a lot of patterns that are not interesting to the users. (b) Setting a large threshold value to the participation index can prune a lot of interesting patterns, or even all of them. (c) Having a uniform threshold value to the participation index for co-occurrence patterns of all frequencies (rare vs. frequent events), and life spans (long-lasting events vs. short-lasting ones) is arguable, since the probability of STCOPs large-size and long-lasting events is inherently smaller than that of co-occurrences of smaller size and short-living patterns. (d) For new areas of spatiotemporal data mining (such as our task of evaluating co-occurrences among solar phenomena), there is simply no background knowledge about the prevalence thresholds that scientists should be using. Therefore, we present here an algorithm that lets the user specify a percentage of most significant (frequent) STCOPs to be mined. The percentage threshold serves only to filter out patterns, while the discovery of knowledge primarily depends on actual data distribution.

Now, we will show the relation between the participation index found for any STCOP using the measures $J$ and $O M A X$. This relation is important, as it will directly influence the number of Top-K\% STCOPs found with the measures $J$ and OMAX, respectively. We will validate this relationship and show the difference in the patterns generated from measures $J$ and $O M A X$ in our experimental section.

LeMma 3.4. For a given user-specified spatiotemporal co-occurrence threshold cce th $_{\text {, }}$ the participation index calculated with measures $J$ and OMAX for any spatiotemporal co-occurrence pattern $\left(S E_{i}\right)$ follows the order $\operatorname{pi}_{J}\left(S E_{i}\right) \leq \operatorname{pi}_{O M A X}\left(S E_{i}\right)$.

Proof. From Lemma 3.1, we know that the selectivity of the measures $J$ and $O M A X$ follows the order $J \leq O M A X$ for any size- $k$ pattern instance for $k \geq 2$.

For a given user-specified threshold $c c e_{t h}$, we denote a participation index $p i\left(S E_{i}\right)$ of a spatiotemporal co-occurrence $S E_{i}$ (see Definition 7), derived from using $J$ as $p i_{J}\left(S E_{i}\right)$, and from using measure $O M A X$ as $\operatorname{piOMAX}\left(S E_{i}\right)$. From Lemma 3.1, we know that the number of pattern instances found for a spatiotemporal co-occurrence $S E_{i}$ follows the order $J \leq O M A X$. Thus, we get that

$$
\begin{gathered}
\min _{j=1}^{k} \operatorname{pr}_{J}\left(S E_{i}, e_{j}\right) \leq \min _{j=1}^{k} \operatorname{pr}_{O M A X}\left(S E_{i}, e_{j}\right) \\
p i_{J}\left(S E_{i}\right) \leq \operatorname{pi}_{O M A X}\left(S E_{i}\right) .
\end{gathered}
$$

In Table III, we show the outputs of classical STCOPs and Top-K\%STCOPs algorithms for the user-sepcified minimum spatiotemporal co-occurrence coefficient (which we will denote as $c c e_{t h}$ ) value 0 . Both approaches use a participation index to discover STCOPs. However, our proposed FastTop-K\%STCOPs algorithm will find only 
Table III. Comparison of Outputs of Classical STCOPs Mining [Ganesan Pillai et al. 2012] and Top-K\%STCOPs for the Example Dataset in Figure 2, with cce $_{\text {th }}=0$

\begin{tabular}{lccccc}
\hline \multirow{2}{*}{$\begin{array}{l}\text { Actual } \\
\text { STCOPs }\end{array}$} & \multicolumn{2}{c}{$\begin{array}{c}\text { Algorithm [Ganesan Pillai et al. } \\
\text { 2012] results }\end{array}$} & \multicolumn{2}{c}{ Top-K\%STCOPs } \\
Algorithm results
\end{tabular}

Note: $\checkmark$ - pattern is in the result set, $\times$ - pattern is pruned.

the patterns that are in the first K\% of the power set (except the empty set and singletons) of the set of all different event types that are based on the highest value of the participation index.

\section{TOP-K\%STCOPS}

In this section, we formally introduce the modeling of Top-K\%STCOPs from datasets with extended spatial representations that evolve over time, and propose algorithms to discover these patterns. Given a set of $M$ spatiotemporal event types, denoted $E=$ $\left\{e_{1}, e_{2}, \ldots, e_{M}\right\}$, and a set of $N$ instances of these event types, which evolves over time, $I=\left\{i_{1}, i_{2}, \ldots, i_{N}\right\}$. A Top-K\%STCOPs is a subset of spatiotemporal event types, whose instances co-occur in both space and time, the volume of these co-occurrences is above the $c c e_{t h}$, and have nonzero $p i$ values among the highest K\% of all STCOPs. Next, we will formalize how the $\mathrm{K} \%$ is defined.

Definition 9. Given $M$ distinct event types, all possible STCOPs represent a powerset of these event types, except the empty set and singletons. Therefore, the total number of STCOPs is equal to $2^{M}-(M+1)$, where $2^{M}$ represents the total number of possible combinations of $M$ event types and $(M+1)$ represents the total number of $M$ singletons plus one for the empty set. The reason we are not interested in reporting the empty set and singletons (i.e., single event types) is because they do not provide any information about spatiotemporal co-occurrences of different event types.

Definition 10. A spatiotemporal co-occurrence pattern is in the Top-K\%STCOPs list when its $p i$ value is among the first K\% of the nonzero pi values of STCOP subsets of the set of all distinct event types, when ordered as a decreasing list based on the values of their participation indices.

For instance, for the dataset shown in Figure 2, if we want to find the Top$30 \% \mathrm{STCOPs}$, only the patterns $e_{1} e_{2}, e_{2} e_{3}$, and $e_{3} e_{4}$ will be in the output list. Similarly, if we want to find the Top-40\%STCOPs, only the patterns $e_{1} e_{2}, e_{1} e_{3}, e_{2} e_{3}, e_{1} e_{2} e_{3}, e_{1} e_{4}$, and $e_{3} e_{4}$ will be in the output list. Note that all three patterns $e_{1} e_{3}, e_{1} e_{2} e_{3}$, and $e_{1} e_{4}$ are included in the output of Top-40\%STCOPs, because they have the same participation index, which causes us to report more than the actual top 40\%. However, this allows us to consistently report all patterns with support reaching top $40 \%$. To distinguish the proposed Top-K\%STCOPs output from the classical spatiotemporal cooccurrence mining algorithm's output [Ganesan Pillai et al. 2012], we show the outputs of Top-30\%STCOPs and Top-40\%STCOPs compared to STCOPs [Ganesan Pillai et al. 2012 ] with a participation index threshold (denoted as $p i_{t h}$ ) value of 0.30 and 0.60 in Table III. 
Definition 11. Given a Top-K\%STCOPs list, and its participation indices, a percentile participation index threshold is the minimum participation index of the TopK\%STCOPs list.

For example, in Table III, for Top- $40 \%$ STCOPs, the percentile participation index threshold is the minimum participation index of patterns $e_{1} e_{2}, e_{1} e_{3}, e_{2} e_{3}, e_{1} e_{2} e_{3}, e_{1} e_{4}$, and $e_{3} e_{4}$, that is, $\min (0.60,0.75,0.50,0.40,0.40,0.40)=0.40$.

Definition 12. A spatiotemporal co-occurring rule of an Top-K\%STCOPs is of the form $S E_{i} \Rightarrow S E_{j}(c c e, K)$, where $S E_{i}$ and $S E_{j}$ are spatiotemporal co-occurrences of different event types, such that $S E_{i} \neq S E_{j}$, and parameters cce and $K$ characterize the rule in the following manner:

(1) cce is an indicator of the strength of the spatiotemporal relation's occurrence that is investigated (in our case, spatiotemporal Overlap);

(2) $K$ is the percentage of all numbers of top STCOPs to be mined.

Definition 13. The conditional probability $\operatorname{cp}\left(S E_{i} \Rightarrow S E_{j}\right)$ of a spatiotemporal cooccurrence rule $S E_{i} \Rightarrow S E_{j}$ is the fraction of pattern instances of $S E_{i}$ that satisfies the spatiotemporal relation strength indicator cce to some pattern instances of $S E_{j}$. It is computed as

$$
\frac{\mid \pi_{S E_{i}}\left(\text { tab_instance }\left(\left\{S E_{i} \cup S E_{j}\right\}\right)\right) \mid}{\mid \text { tab_instance }\left(\left\{S E_{i}\right\}\right) \mid},
$$

where $\pi$ is the relational projection operation with duplicate elimination [Huang et al. $2004]$.

For example, for co-occurrence rule $e_{1} \Rightarrow e_{2}$, from our dataset in Figure 2 and Table I, the conditional probability is equal to

$$
\frac{\mid \pi_{e_{1}}\left(\text { tab_instance }\left(e_{1} e_{2}\right)\right) \mid}{\mid \text { tab_instance }\left(e_{1}\right) \mid}=3 / 5=60 \% .
$$

In other words, only three out of five instances $\left(i_{1}, i_{2}, i_{4}\right)$ of event type $e_{1}$ co-occur with instances $\left(i_{7}, i_{6}\right.$, and $\left.i_{8}\right)$ of event type $e_{2}$. The conditional probability is used to compute the confidence of rules derived from a Top-K\%STCOP pattern. We report only the rules of Top-K\%STCOP pattern that satisfy the conditional probability threshold.

\subsection{Problem Statement}

\section{Given}

(1) A set of spatiotemporal event types $E=\left\{e_{1}, e_{2}, \ldots, e_{M}\right\}$ over a common spatiotemporal framework.

(2) A set of $N$ event instances $I=\left\{i_{1}, i_{2}, \ldots, i_{N}\right\}$, which evolve over time and space such that $M \ll N$, and each $i_{j} \in I$ is a tuple <instance-id, spatiotemporal event type, sequence of $<2 D$ shape, matching time instant $>$ pairs $>$, where the sequence of $2 D$ shape and matching time-instant pairs reflects the evolution of the spatiotemporal event.

(3) A user-specified spatiotemporal co-occurrence coefficient threshold $\left(\right.$ cce $\left._{t h}\right)$.

(4) A user-specified percentage of number of top STCOPs to be mined (K).

(5) A user-specified conditional probability threshold $\left(c p_{t h}\right)$.

(6) A time interval of temporal data sampling, denoted $\Delta t$. For simplicity, we assume that all events are sampled with the same time interval, making the shapes of individual events exactly aligned in time. 
Овлестіve. Find the complete and correct result set that is in the first K\% of STCOPs subset and the corresponding rules based on the highest value of participation index ( $p i>p i_{t h}$ with cce $>c c e_{t h}$ and $\left.c p>c p_{t h}\right)$.

\subsection{Mining Top-K\%STCOPs}

In this section, we first provide a naïve approach to discover Top-K\%STCOPs, then provide a computationally efficient novel algorithm called the FastTop-K\%STCOPs algorithm to discover Top-K\%STCOPs. We also provide an execution trace of our FastTop-K\%STCOPs algorithm for the dataset shown in Figure 2, focusing on mining Top-K\%STCOPs.

4.2.1. Naïve Approach. A Naïve Top-K\%STCOPs approach can use our original spatiotemporal co-occurrence mining algorithm [Ganesan Pillai et al. 2012] to find all the STCOPs, then run a post-processing step to find Top-K\%STCOPs. This approach will generate all possible STCOPs for the given $c c e_{t h}$ and $p i_{t h}$ values, then apply a pruning step at the end to determine the at most Top-K\%STCOPs while ordered based on their $\mathrm{cp}$. This approach, however, leads to unnecessary computational cost since non-Top-K\%STCOPs are not pruned before the post-processing step.

4.2.2. FastTop-K\%STCOPs Algorithm. To improve the Naïve Top-K\%STCOPs approach, we propose a novel FastTop-K\%STCOPs mining algorithm by including a filtering step in each iteration of the algorithm. The FastTop-K\%STCOPs algorithm maintains a candidate list of the Top-K\% at each iteration of the algorithm (see Definition 10). The FastTop-K\%STCOPs algorithm uses this candidate list of the Top-K\% to discover the correct Top-K\%STCOPs. The percentile participation index threshold is the minimum participation index of the STCOPs of the Top-K\% of the candidate list (see Definition 11). If a newly generated pattern has a participation index value greater than the percentile participation index, and there is no other pattern in the candidate list with the same participation index as the newly generated pattern, then the newly generated pattern will replace the pattern that has the minimum participation index in the Top-K\% candidate list. However, if a newly generated pattern has a participation index value greater than the percentile participation index and there are pattern(s) in the candidate list with the same participation index as the newly generated pattern, then the newly generated pattern will be added to the Top-K\% candidate list. Also, if a newly generated pattern has a participation index value equal to the percentile participation index, then the newly generated pattern will be added to the Top-K\% candidate list. In Figure 4, we give the pseudo-code of the proposed FastTop-K\%STCOPs algorithm; then, we provide a execution trace of the FastTop-K\%STCOPs algorithm using the dataset shown in Figure 2.

In Figure 4, the inputs are as defined in Section 4.1. In the algorithm, Steps 1 and 2 initialize the parameters and data structures, Steps 3 through 8 give an iterative process to discover the Top-K\%STCOPs, and Step 9 finds Top-K\%STCOPs by filtering STCOPs whose participation index value is equal to zero. Step 10 generates rules from the Top-K\%STCOPs found in Step 9 and the algorithm returns Top-K\%STCOPs and the corresponding rules in Step 11.

Generation of table instances of size-1 (Step 2): In this function, argument $\Delta t$ represents the increment in the number of timesteps. The movement of an instance from its start and end time slot is projected using $\Delta t$ (to increment the number of timesteps between time slots). The combination of the event instance ID and timestep will allow us to identify an event at a particular moment.

Generation of candidate co-occurrence patterns (Step 4): This function uses an Apriori-based approach to generate candidates of size- $(k+1)$ using size- $k$ Top-K\% 


\section{Inputs:}

(I1) $E=$ A set of spatiotemporal event types.

(I2) A set of instances of these event types, $I=<$ instance-id, spatiotemporal event type, sequence of $2 D$ shape and matching time instant pairs $>$.

(I3) User-specified minimum spatiotemporal co-occurrence coefficient threshold cce $_{t h}$ (see Definition 4).

(I4) User-specified percentage of number of STCOPs to be mined K (see Definition 10).

(I5) User-specified minimum conditional probability threshold $c p_{t h}$ (see Definition 13).

(I6) A user-specified sampling interval $(\Delta t)$, measured as duration between snapshots of evolving objects.

\section{Output:}

At the most Top-K\%STCOPs and the corresponding rules with cce greater than the user-specified minimum threshold value given on input.

\section{Variables:}

(V1) $k$ the co-occurrence size (see Definition 1).

$(V 2) C_{k}$ : a set of candidates for size- $k$ STCOPs derived from size- $(k-1)$

Top-K\%STCOPs.

(V3) $T_{k}$ : set of tab_instance of size- $k S E_{i}$ (see Definition 3).

(V4) $P_{k}$ : a set of size- $k$ Top-K\%STCOPs derived from size- $k$ candidate Top-K\%STCOPs.

(V5) Cand_list: sets of Top-K\%STCOPs.

(V6) AtMost_Top-K\%: sets of Top-K\%STCOPs.

(V7) $R$ : a set of spatiotemporal co-occurrence rules derived from Top-K\%STCOPs.

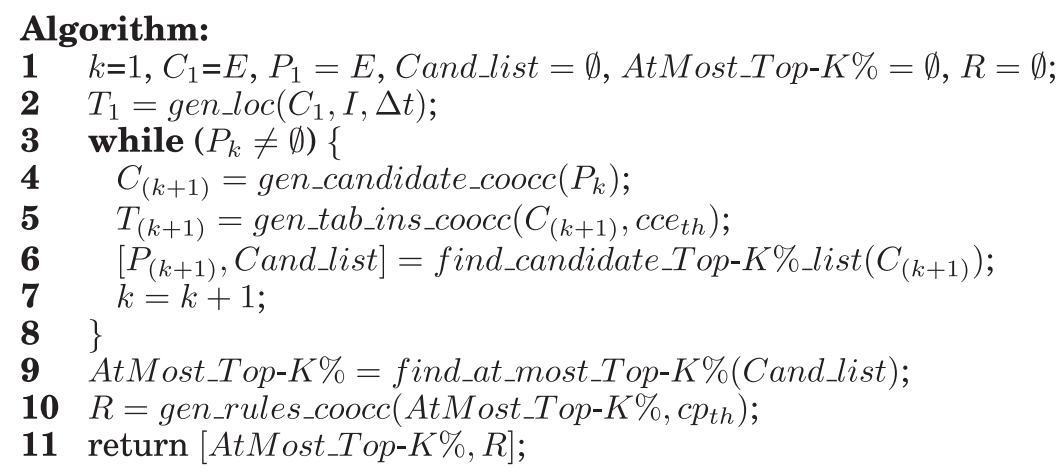

Fig. 4. FastTop-K\%STCOPs algorithm.

patterns when $k>1$; otherwise, the function generates candidates of size-2 using spatiotemporal event types $(E)$.

Generation of table instances of size-(k+1) (Step 5): This function is similar to the table instance for candidates' co-occurrence patterns in the spatiotemporal co-occurrence mining algorithm. However, this method takes size- $(k+1)$ candidate Top-K\%STCOPs when $k>1$. Pattern instances for each table instance can be generated by a spatiotemporal query. The geometric shapes of the instances at each timestep are saved, as these geometric shapes will be used for finding the cce of STCOPs of size three or more. In this function, we calculate the cce for each pattern instance by using measures $J$ or OMAX. Pattern instances that have a cce below the user-specified cce $_{t h}$ value are deleted from the table instance. 
Find candidate Top-K\%STCOPs list (Step 6): In this step, we maintain the Top-K\%STCOPs candidate list and generate size- $(k+1)$ patterns using size- $k$ Top-K\% patterns. Initially, the function selects size-2 Top-K\%STCOPs out of all STCOP subsets (see Definition 10), and saves them in the Top-K\% candidate list. The percentile participation index threshold (see Definition 11) is calculated by finding the minimum participation index of the STCOPs of the Top-K\% of the candidate list. Using the size-2 Top-K\%STCOPs, the function generates size-3 patterns. If a newly generated size-3 pattern has a participation index value greater than the percentile participation index threshold and there are no other size-2 patterns of the same participation index as the newly generated size-3 pattern in the candidate list, then the newly generated size-3 pattern will replace the pattern with percentile participation index in the candidate list. However, if a newly generated size-3 pattern has a participation index value greater than the percentile participation index threshold and there are size-2 patterns of the same participation index as the newly generated size-3 pattern in the candidate list, then the newly generated size-3 pattern will be added to the Top-K\% candidate list. Moreover, if a newly generated size-3 pattern has a participation index value equal to the percentile participation index, it will be added to the Top-K\% candidate list. Similarly, size-4 patterns will be generated using size-3 Top-K\%STCOPs, and the Top-K\% candidate list will be updated accordingly. The algorithm runs iteratively until no more candidate STCOPs can be generated. The candidate list contains the union of all sizes of Top-K\%STCOPs.

Find Top-K\%STCOPs (Step 9): This function outputs a list with Top-K\%STCOPs obtained from the candidate list.

Generate rules from the Top-K\%STCOPs (Step 10): In this function, we generate spatiotemporal co-occurrence rules. A set of spatiotemporal co-occurrence rules $R$ are generated from the Top-K\%STCOPs generated in Step 9. The conditional probability (Definition 14) $c p\left(S E_{i} \Rightarrow S E_{j}\right)$ of a spatiotemporal co-occurrence rule $S E_{i} \Rightarrow S E_{j}$ is calculated using Equation (13). Finally, the algorithm returns Top-K\%STCOPs, and the corresponding spatiotemporal co-occurrence rules that satisfy the user-specified minimum conditional probability threshold $c p_{t h}$ in Step 11.

\subsubsection{Correctness and Completeness Analysis of the FastTop-K\%STCOPs.}

\section{Theorem 4.2.3.1. The FastTop-K\%STCOPs is complete.}

Proof. The FastTop-K\%STCOPs is complete if it finds Top-K\%STCOPs out of all STCOP subsets defined in Definition 9. First, the algorithm will find all first K\% of the nonzero $p i$ values of STCOP subsets of the set of all distinct event types based on the highest value of their participation indices (Definition 10). We show this by proving that none of the functions in the proposed algorithm misses any patterns.

The gen_candidate_coocc function does not miss any patterns given the antimonotone nature of the participation index. The input to this function is candidate size- $k$ top-K\% STCOPs and the output is candidate size- $(k+1)$ STCOPs.

The gen_tab_ins_coocc function does not miss any patterns. In this function, pattern instances of candidate size- $(k+1)$ are generated by joining the pattern instances of size- $k$ top-K\% STCOPs if they satisfy the co-occurrence threshold $c c e_{t h}$.

The find_candidate_Top-K\% list function does not miss any patterns. This function determines the Top-K\%STCOPs candidate list using the percentile participation index. In this function, the newly generated size- $(k+1)$ that has a participation index value greater than the percentile participation index threshold will replace the pattern that has the percentile participation index in the candidate list only when there are no other patterns in the candidate list with the same participation index as the newly 
generated size- $(k+1)$. In other cases, the newly generated size- $(k+1)$ will be added only to the candidate list (see Step 6 in Section 4.2.2).

\section{TheOREM 4.2.3.2. The FastTop-K\%STCOPs is correct.}

Proof. This proof is established based on the pruning steps of find_candidate_Top $K \%$ list, which weed out candidates not found in the top-K\% STCOP list. An STCOP is in the Top-K\%STCOPs list when its $p i$ value is among the first $\mathrm{K} \%$ of the nonzero $p i$ values of STCOP subsets of the set of all distinct event types based on the highest value of their participation indices (Definition 10). In the find_candidate_Top-K\%_list function, if we find a tie condition in the Top-K\%STCOPs candidate list, we will include more than the Top-K\% patterns until there is no tie. Thus, we will not miss any TopK\%STCOPs.

Next, we attempt to clarify the conditions necessary for pruning to happen and the factors that affect the amount of pruning. The naïve approach does not prune out non-topK\% patterns before the post-processing step. However, the proposed FastTopK\%STCOPs approach uses a filtering step to incorporate pruning at each iteration of the algorithm. In particular, the impact of pruning in the FastTop-K\%STCOPs approach will be more effective when the number of nontop-K\% patterns is higher in the dataset. For example, on the one hand, when we attempt to derive top-100\%STCOPs from the spatiotemporal dataset shown in Figure 2, we need to mine the 11 highest participation index patterns; thus, pruning will not be effective since we are interested in finding $100 \%$ of the 11 STCOP subsets. On the other hand, when we attempt to derive top$40 \%$ STCOPs, the pruning will be more effective since we will be filtering out a lot of nontop-K\% patterns in the filtration step of the algorithm. Moreover, the impact of pruning will be more effective on datasets with many event types, since the increase in the number of event types can cause an increase in the number of join operations, which is computationally expensive.

Execution trace: Here, we present an execution trace of the algorithm, focusing on mining Top-K\%STCOPs. Our example dataset contains four different event types $e_{1}, e_{2}, e_{3}$, and $e_{4}$ (see Section 3.2). The total number of STCOP subsets of these event types is 11 (as can be derived from Definition 9). For example, if we want to find the Top-40\%STCOPs from the spatiotemporal dataset shown in Figure 2, we need to mine the 4 highest participation index patterns (i.e., $40 \%$ of the 11 STCOP subsets). The candidate patterns for size-2 and the corresponding table instances are generated in Steps 4 and 5. The next step is to find the size-2 Top-40\%STCOPs candidate list and the percentile participation index from the generated candidate list. The size-2 Top$40 \% \mathrm{STCOPs}$ candidate list will include patterns $e_{1} e_{2}, e_{1} e_{3}, e_{2} e_{3}, e_{1} e_{4}$, and $e_{3} e_{4}$ (see Table III). The percentile participation index calculated from the minimum participation index of patterns is $\min (0.60,0.40,0.75,0.40,0.50)=0.40$. These size- 2 patterns will be used to generate candidate patterns of size-3. The size-3 patterns generated will be $e_{1} e_{2} e_{3}$, and the participation index value is 0.40 (see Table III). The next step is to determine whether the Top- $40 \%$ candidate list and percentile participation need to be updated based on the newly generated size-3 patterns. The pattern $e_{1} e_{2} e_{3}$ will be included in the Top- $40 \%$ candidate list, since its participation index value is equal to the percentile participation index threshold value. In the next iteration, the algorithm will not generate size-4 patterns since there are not enough subsets of size-3 to make it possible (e.g., we are missing patterns $e_{1} e_{2} e_{4}, e_{2} e_{3} e_{4}$, and $e_{1} e_{3} e_{4}$, which are necessary to even consider whether pattern $e_{1} e_{2} e_{3} e_{4}$ is possible). Thus, the output of the algorithm will be $e_{1} e_{2}, e_{1} e_{3}, e_{2} e_{3}, e_{1} e_{4}, e_{3} e_{4}$, and $e_{1} e_{2} e_{3}$ and the rules generated from these patterns. 


\section{EXPERIMENTAL EVALUATION}

In this section, we evaluate our FastTop-K\%STCOPs algorithm and the Naïve Top$\mathrm{K} \%$ STCOPs algorithm on two different measures (used to evaluate our cce): Jaccard (J) and $O M A X$. We use three real-life datasets from the solar physics domain. Specifically, we evaluate our algorithms using six types of evolving solar phenomena $(|E|=6)$. We report results based on performance and impact of our measures on capturing the different spatial and temporal characteristics of the real-life spatiotemporal dataset, using FastTop-K\%STCOPs and the Naïve Top-K\%STCOPs algorithms. All experiments were performed using PostgreSQL 9.1.4 and PostGIS 1.5.4.

\subsection{Real-Life Dataset}

Our real-life datasets contain evolving instances of six different solar event types, which were observed using the SDO telescope during 01/01/2012 - 01/01/2012 (dataset $A$ ), 01/01/2012 - 01/03/2012 (dataset B), and 01/01/2012 - 01/05/2012 (data set $C$ ). We obtained our datasets from the well-known solar data repository called the Heliophysics Event Knowledgebase (HEK) [HEK 2013; Hurlburt et al. 2010; Schuh et al. 2013]. The six different solar event types in our dataset are: Active Region, Filament, Sigmoid, Sunspot, Flare, and Emerging Flux. For our experiments, we denote Active Region (labelled e1), Filament (e2), Sigmoid (e3), Sunspot (e4), Flare (e5), and Emerging Flux (e6). For dataset A, differential rotation [Howard et al. 1990] of the sun is used to estimate an approximate location for our next detection of the same instance at a later time. For datasets $B$ and $C$, we used the multiple-target tracking approach proposed in Kempton et al. [2014] to track the instances of various solar event types. All of our datasets are available on-line to let researchers interested in this topic reproduce our experiments. The website for this article can be found at Ganesan Pillai [2014a]. We report results based on performance and impact of our measures on capturing the different spatial and temporal characteristics of the real-life spatiotemporal dataset, using FastTop-K\%STCOPs and the Naïve Top-K\%STCOPs algorithms. For all our datasets, cce $_{t h}$ values were set to 0.01 , and the sampling time interval $\Delta t$ were set at $30 \mathrm{~min}$. For dataset $A$, the percentage of number of STCOPs K was set at 15 and the conditional probability threshold $c p_{t h}$ (see Definition 13) was set at 0.60 . For dataset $B$, the percentage of number of STCOPs K was set at 20 and the conditional probability threshold $c p_{t h}$ was set at 0.70 . For dataset $C$, the percentage of number of STCOPs K was set at 25 and the conditional probability threshold $c p_{t h}$ was set at 0.70 . Recall from Sections 3 and 4, for our proposed FastTOP-K\%STCOPs, the number of STCOPs generated depends on the values of two parameters: $c c e_{t h}$ and the K. The $c c e_{t h}$ threshold is used to assess the strength of the spatiotemporal relation Overlap and the $\mathrm{K}$ threshold is used to determine the percentage of all numbers of top STCOPs to be mined. $c p_{t h}$ is used during the final rule-generation phase and sets an upper bound on the selected rules. The default value of $c c e_{t h}$ threshold is chosen to be a very low value in our experiments so that we discover as many pattern instances as possible from datasets with different spatial and temporal characteristics. In certain cases, even a small increase in $c c e_{t h}$ may filter out a lot of patterns if the behavior of our data is such that lots of patterns co-occur with a minor Overlap value (e.g., they co-occur for a brief amount of time, spatially overlapping only a little-as colliding particles in physics that spatially overlap only for fractions of a second), and very few with high values of their Overlap (e.g., they co-exist closely overlapping in space through their common life-times-like life-long symbiosis between certain biological species). We choose these $\mathrm{K}$ and $c p_{t h}$ values in order to be able to analyze the effects of pruning and impact of our measures on datasets with different spatiotemporal characteristics. In our experiments, we varied the percentage of number of STCOPs $\mathrm{K}$ and the 
conditional probability threshold $c p_{t h}$ across the three datasets with different spatiotemporal characteristics. Note that, in experimental analysis, we do not show the extensive comparison of FastTOP-K\%STCOPs and naïve approaches by changing the percentage of STCOPs K (and conditional probability threshold $c p_{t h}$ ) in any single dataset; this setup is not intended to extensively show how big the impact of the chosen thresholds is on number of discovered patterns, as these depend on assigned thresholds as well as on the statistical characteristics of the data (e.g., how big events are, how quickly they evolve, how many were present, and so on). Instead, we show the pruning effect of FastTOP-K\%STCOPs. The pruning effect of our proposed FastTop-K\%STCOPs is expected to outperform the naïve approach when the percentage of STCOPs K is small in comparison to the total number of all possible STCOPs (see Definition 9). For example, when $\mathrm{K}$ is 100 , the proposed early pruning strategy of FastTOP-K\%STCOPs will not be effective, since we will be required to find all possible STCOPs. For smaller K values, the naïve approach will suffer from high computational cost involved in finding the nontop-K\% patterns. Since the number of discovered patterns is dependent on frequencies of actual co-occurrences in the data, there is not much in terms of heuristics that we can propose for the right choices of $c c e_{t h}$ and $\mathrm{K}$ values. This is a problem common to all frequent pattern-mining algorithms, starting from shopping basket data, bio-sequence data, and ending at frequent subgraph mining. The only dependency that we can guarantee to hold for region-based spatiotemporal data is that, as we increase $c c e_{t h}$ and decrease $\mathrm{K}$, our proposed FastTOP-K\%STCOPs algorithm will not run slower than in the naïve alternative. This is because, independent of dataset characteristics, with these conditions changed, the number of generated patterns can only decrease or remain the same. This is what most frequent pattern-mining experts describe as "monotonically nonincreasing dependency."

\subsection{Memory Usage}

We first investigated the memory usage of the FastTop-K\%STCOPs and Naïve TopK\%STCOPs algorithms for the candidate table instances generated. We report the hard-drive memory usage of candidate table instances with all the pattern instances generated, and memory usage of candidate table instances after filtering the pattern instances that do not satisfy threshold $c c e_{t h}$.

In Figure 5, we show the memory usage of table instances used by the FastTopK\%STCOPs and Naïve Top-K\%STCOPs algorithms for different pattern sizes. For datasets $A, B$, and $C$ in Figure 5(a), the dotted bar represents the memory usage of table instances for all pattern instances generated (i.e., the candidates), and the white bar represents the memory usage of table instances after filtering out the pattern instances that do not satisfy the threshold $c c e_{t h}$ with measure $J$ in the Naïve TopK\%STCOPs algorithm (i.e., our actual patterns). Similarly, for datasets $A, B$, and $C$ in Figure 5(a), the bricked bar represents the memory usage of table instances for all pattern instances generated (i.e., the candidates), and the crossed bar represents the memory usage of table instances after filtering out the pattern instances that do not satisfy the threshold $c c e_{t h}$ with measure $J$ in the FastTop-K\%STCOPs algorithm.

As expected, for all three datasets from Figure 5(a), we can observe that there is a drop in the memory usage after the pattern instances are filtered by applying the threshold cce $_{t h}$ (compare Naïve Top-K\%STCOPs (J-BeforeCCE) with Naïve Top-K\%STCOPs (J-AfterCCE), and FastTop-K\%STCOPs (J-BeforeCCE) with FastTopK\%STCOPs (J-AfterCCE) in Figure 5(a)). However, generation of all J-BeforeCCEs is neccessary to discover actual spatiotemporal co-occurrence patterns (i.e., our JAfterCCEs). A similar argument holds for the measure $O M A X$, as seen in Figure 5(b). Moreover, the memory usage of FastTop-K\%STCOPs algorithm with measures $J$ and $O M A X$ is less than the memory usage of the Naïve Top-K\%STCOPs algorithm. This 


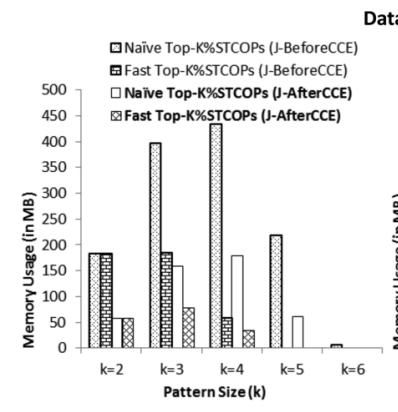

(a) Measure $J$

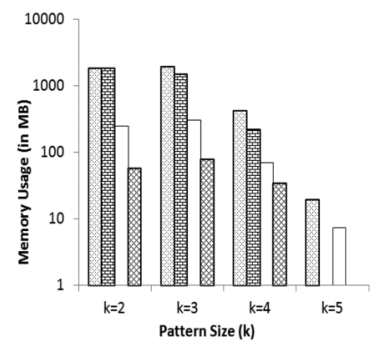

(a) Measure J

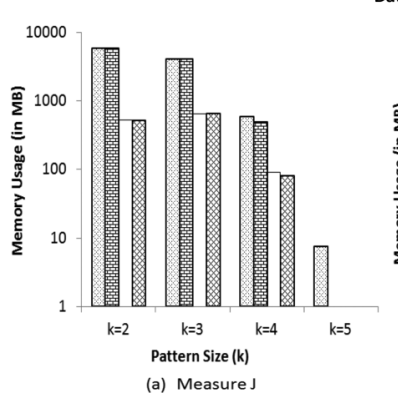

Dataset A

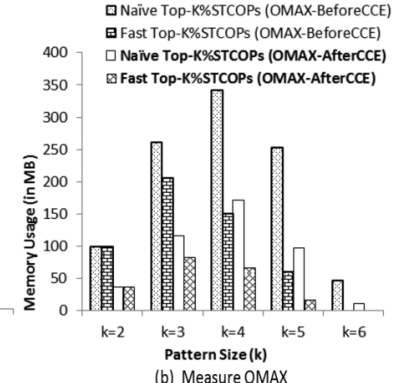

Dataset B

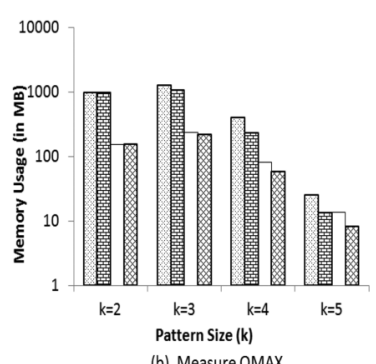

Dataset C

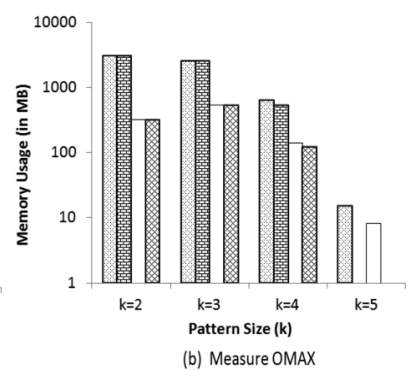

Fig. 5. Memory usage used by candidate table instances for the FastTop-K\%STCOPs and Naïve TopK\%STCOPs algorithms. Note that we report on dataset A using a linear scale, while for datasets B and $\mathrm{C}$ (much bigger data), we had to use log scales.

is due to the pruning strategy that we use in our FastTop-K\%STCOPs (see Step 6 of our FastTop-K\%STCOPs algorithm). Furthermore, the memory usage with measure $J$ is more expensive than the memory usage with measure $O M A X$. This is due to the fact that, in order to calculate the measure $J$, we require union geometries of the pattern instances. Thus, for both the FastTop-K\%STCOPs and Naïve Top-K\%STCOPs algorithms, the measure $J$ consumes more memory than the measure OMAX.

\subsection{Execution Time}

Next, we analyzed the execution time of our FastTop-K\%STCOPs and Naïve TopK\%STCOPs algorithms. For datasets $A, B$, and $C$, we show in Figure 6(a) and 6(b) the execution times for patterns of different sizes for the measures $J$ and OMAX, respectively. In Figure 6, the white bar represents the execution time of Naïve TopK\%STCOPs for different pattern sizes, and the crossed bar represents the execution time of FastTop-K\%STCOPs for different pattern sizes. As expected, our FastTopK\%STCOPs algorithm outperforms the Naïve Top-K\%STCOPs algorithm, since it uses 


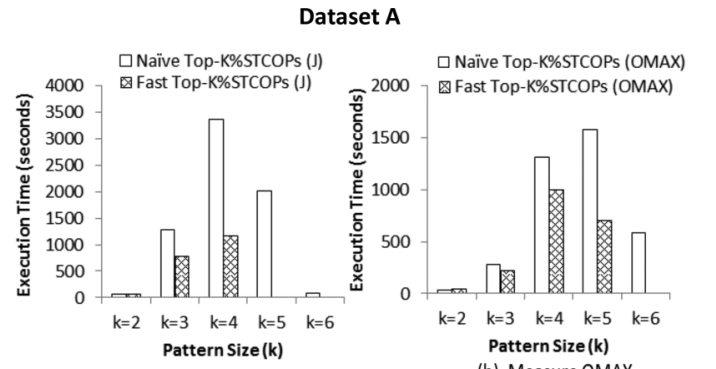

(a) Measure J

(b) Measure OMAX

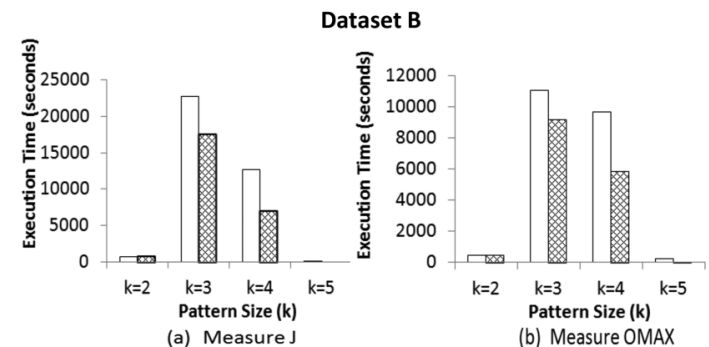

Dataset C
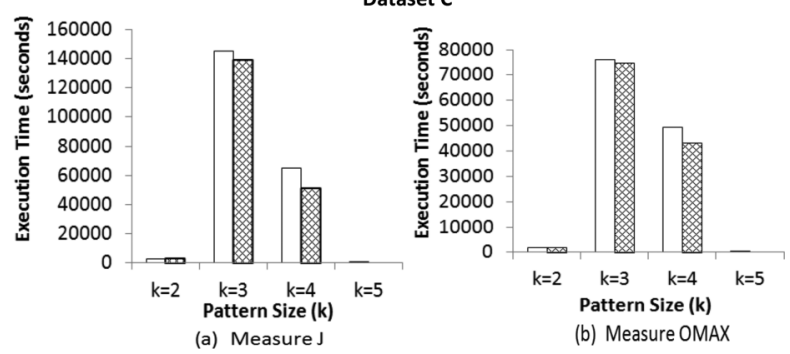

Fig. 6. Camparison of execution time for the FastTop-K\%STCOPs and Naïve Top-K\%STCOPs algorithms.

a pruning strategy to find candidate patterns (see Step 6 of our FastTop-K\%STCOPs algorithm). On the other hand, the Naïve Top-K\%STCOPs algorithm generates all patterns, then finally uses a post-processing step to discover Top-K\%STCOPs, thus resulting in longer execution time. Moreover, in Figure 6(a) and (b), we can observe that the execution time of both FastTop-K\%STCOPs and Naïve Top-K\%STCOPs algorithms is more expensive with measure $J$ than with measure $O M A X$. Similar to the argument that we presented in memory usage, this is again because of the fact that the measure $J$ requires expensive union geometries. This memory overhead causes the execution time of both the Naïve Top-K\%STCOPs and the FastTop-K\%STCOPs algorithms to be slower with measure $J$ in comparison to the measure OMAX. Also, note that as we increase the percentage of STCOPs $\mathrm{K}$ for datasets $A, B$, and $C$, the difference in execution time between Naïve Top-K\%STCOPs and the FastTop-K\%STCOPs algorithms for different pattern sizes is reduced. This is due to the fact that as we increase the percentage of STCOPs K FastTop-K\%STCOPs, the algorithm cannot prune many candidates. Moreover, from Figures 5 and 6, moving from pattern size $k=2, k=3$, and $k=4$, we can clearly see a transition of the algorithm, moving from data/storage to computational intensive. For $k=2$, we see a lot of memory usage, but relatively small runtime, probably because spatial joins (ST Overlaps) are calculated only for pairs of objects. For $k=3$, the machine struggles, having a lot of joins to compute, for $k=4$ memory usage decreases while computational intensiveness remains. 


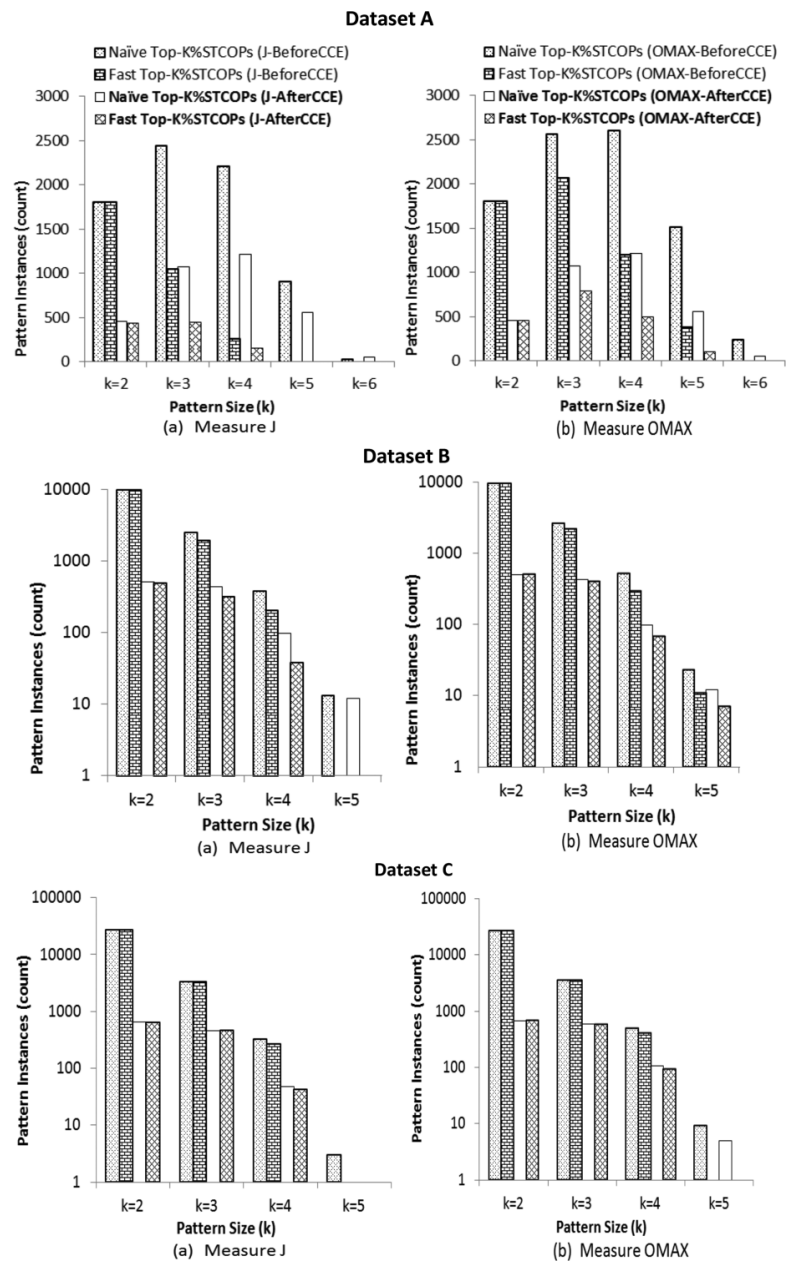

Fig. 7. Number of pattern instances generated by the FastTop-K\%STCOPs and Naïve Top-K\%STCOPs algorithms. Note that we report on dataset A using a linear scale, while for datasets B and C (much bigger data), we had to use log scales.

\subsection{Pattern Instances}

For datasets $A, B$, and $C$ in Figure 7(a) and 7(b), we show the count of pattern instances generated by the FastTop-K\%STCOPs and Naïve Top-K\%STCOPs algorithms for different pattern sizes with measures $J$ and $O M A X$, respectively. For datasets $A, B$, and $C$ in Figure 7(a), the dotted bar represents the count of all pattern instances generated (i.e., the candidates), and the white bar represents the count of pattern instances after filtering out the pattern instances that do not satisfy the threshold $c c e_{t h}$ with measure $J$ in the Naïve Top-K\%STCOPs algorithm (i.e., our actual patterns). Similarly, in Figure 7(a), the bricked bar represents the count of all the pattern instances generated (i.e., the candidates), and the crossed bar represents the count of pattern instances after filtering out the pattern instances that do not satisfy the threshold $c c e_{t h}$ with measure $J$ in the FastTop-K\%STCOPs algorithm. As expected, from Figure 7(a), we can observe that there is a drop in the count of pattern instances after applying the threshold $c c e_{t h}$ (compare Naïve Top-K\%STCOPs (J-BeforeCCE) with Naïve Top-K\%STCOPs 


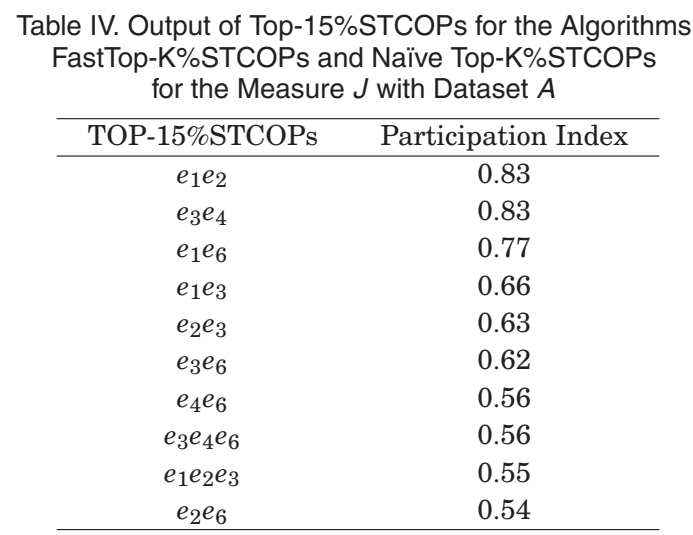

\begin{tabular}{cc} 
Table V. Output of Top-15\%STCOPs for the Algorithms \\
FastTop-K\%STCOPs and Naïve Top-K\%STCOPs \\
for the Measure OMAX with Dataset $A$ \\
\hline TOP-15\%STCOPs & Participation Index \\
\hline$e_{1} e_{2}$ & 0.83 \\
$e_{1} e_{6}$ & 0.83 \\
$e_{3} e_{4}$ & 0.83 \\
$e_{1} e_{3}$ & 0.66 \\
$e_{2} e_{3}$ & 0.63 \\
$e_{2} e_{6}$ & 0.63 \\
$e_{3} e_{6}$ & 0.82 \\
$e_{3} e_{4} e_{6}$ & 0.56 \\
$e_{4} e_{6}$ & 0.56 \\
$e_{1} e_{2} e_{3}$ & 0.55 \\
$e_{1} e_{2} e_{6}$ & 0.54 \\
$e_{1} e_{5}$ & 0.50 \\
$e_{1} e_{3} e_{6}$ & 0.50 \\
\hline
\end{tabular}

(J-AfterCCE), and FastTop-K\%STCOPs (J-BeforeCCE) with FastTop-K\%STCOPs (JAfterCCE) in Figure 7(a)). However, generation of all J-BeforeCCEs is neccessary to discover actual spatiotemporal co-occurrence patterns (i.e., our J-AfterCCEs). Similarly, the argument holds for the measure $O M A X$, as seen in Figure 7(b). Moreover, the count of pattern instances of the FastTop-K\%STCOPs algorithm with measures $J$ and $O M A X$ is less than the count of pattern instances of the Naïve Top-K\%STCOPs algorithm. This is due to the pruning strategy that we use in our FastTop-K\%STCOPs (see Step 6 of our FastTop-K\%STCOPs algorithm). Furthermore, the count of pattern instances with measure $J$ is less than the count of pattern instances with measure $O M A X$. This is due to the fact that the selectivity of measures follows the order $J \leq O M A X$, as shown in Lemma 3.1. Thus, for both the FastTop-K\%STCOPs and Naïve Top-K\%STCOPs algorithms, the measure $J$ generates fewer pattern instances than the measure $O M A X$.

\subsection{Discussion on Top-K\%STCOPs for the Measures $J$ and OMAX}

In this section, we analyze the Top-K\% STCOPs generated from the measures $J$ and $O M A X$ from both our FastTop-K\%STCOPs and Naïve Top-K\%STCOPs algorithms for dataset $A$. However, these arguments hold for datasets $B$ and $C$ as well. Tables IV and $\mathrm{V}$ show the Top-K\% STCOPs discovered using the measures $J$ and $O M A X$, respectively. From Table IV, we can observe that there are 10 patterns discovered using the measure 
Table VI. Output of Rules Generated from Top-15\%STCOPs for the Algorithms FastTop-K\%STCOPs and Naïve Top-K\%STCOPs for the Measure $J$ with Dataset $A$

\begin{tabular}{lcc}
\hline TOP-15\%STCOPs & Co-occurrence Rule & Conditional Probability \\
\hline \multirow{2}{*}{$e_{1} e_{2}$} & $e_{2} \Rightarrow e_{1} e_{2}$ & 1.00 \\
& $e_{1} \Rightarrow e_{1} e_{2}$ & 0.83 \\
$e_{3} e_{4}$ & $e_{4} \Rightarrow e_{3} e_{4}$ & 1.00 \\
& $e_{3} \Rightarrow e_{3} e_{4}$ & 0.83 \\
$e_{1} e_{6}$ & $e_{6} \Rightarrow e_{1} e_{6}$ & 0.93 \\
& $e_{1} \Rightarrow e_{1} e_{6}$ & 0.77 \\
$e_{1} e_{3}$ & $e_{3} \Rightarrow e_{1} e_{3}$ & 1.00 \\
& $e_{1} \Rightarrow e_{1} e_{3}$ & 0.66 \\
$e_{2} e_{3}$ & $e_{3} \Rightarrow e_{2} e_{3}$ & 1.00 \\
& $e_{2} \Rightarrow e_{2} e_{3}$ & 0.63 \\
$e_{3} e_{6}$ & $e_{3} \Rightarrow e_{3} e_{6}$ & 0.83 \\
$e_{4} e_{6}$ & $e_{6} \Rightarrow e_{3} e_{6}$ & 0.62 \\
& $e_{4} \Rightarrow e_{4} e_{6}$ & 1.00 \\
& $e_{4} \Rightarrow e_{3} e_{4} e_{6}$ & 1.00 \\
$e_{3} e_{4} e_{6}$ & $e_{3} e_{4} \Rightarrow e_{3} e_{4} e_{6}$ & 1.00 \\
& $e_{4} e_{6} \Rightarrow e_{3} e_{4} e_{6}$ & 1.00 \\
& $e_{3} e_{6} \Rightarrow e_{3} e_{4} e_{6}$ & 0.92 \\
& $e_{3} \Rightarrow e_{3} e_{4} e_{6}$ & 0.83 \\
& $e_{3} \Rightarrow e_{1} e_{2} e_{3}$ & 1.00 \\
$e_{1} e_{2} e_{3}$ & $e_{2} e_{3} \Rightarrow e_{1} e_{2} e_{3}$ & 0.87 \\
& $e_{1} e_{3} \Rightarrow e_{1} e_{2} e_{3}$ & 0.74 \\
$e_{2} e_{6}$ & $e_{2} \Rightarrow e_{1} e_{2} e_{3}$ & 0.63 \\
& $e_{6} \Rightarrow e_{2} e_{6}$ & 0.75 \\
\hline
\end{tabular}

$J$; however, from Table $\mathrm{V}$, we can observe that there are 13 patterns discovered using the measure OMAX. From Lemma 3.1, we know that, for a pattern instance, the selectivity of the measures $J$ and $O M A X$ follows the order $J \leq O M A X$. From Lemma 3.4, we know that, for a spatiotemporal co-occurrence pattern $\left(S E_{i}\right)$, the participation index of the pattern $S E_{i}$ follows the order $p i_{J}\left(S E_{i}\right) \leq p i_{O M A X}\left(S E_{i}\right)$ for the measures $J$ and $O M A X$. However, if we sort all the STCOPs generated based on participation index, the patterns generated from measures $J$ and $O M A X$ might result in different ordering. For example, see the pattern $e_{2} e_{6}$ in Tables IV and V. Note that this is due to selectivity of the measures $J$ and OMAX (see Lemma 3.1) and the selectivity influence on the participation index derived from the measures $J$ and $O M A X$ for a pattern $S E_{i}$ (see Lemma 3.4). Thus, we might get a different number of Top-K\%STCOPs derived from the measures $J$ and $O M A X$. Moreover, the results from Table IV and V validate our implementation of our FastTop-K\%STCOPs algorithm.

\subsection{Discussion on Co-occurrence Rules Generated from Top-K\%STCOPs for the Measures $J$ and OMAX}

Finally, in Tables VI and VII, we investigate the rules generated from Top-K\%STCOPs discovered from the measures $J$ and $O M A X$, respectively, for the dataset $A$. Note that the confidence of co-occurrence rules are calculated using the conditional probability (Definition 13). The selectivity of the measures directly influences the confidence of a co-occurrence rule. The importance of analyzing different measures is shown here in order to accurately capture the spatiotemporal characteristics of different solar events. For instance, $J$ gives much lower values to objects that have a small Intersection volume, giving a penalty to some of our events that are small in area and 
Table VII. Output of Rules Generated from Top-15\%STCOPs for the Algorithms FastTop-K\%STCOPs and Naïve Top-K\%STCOPs for the Measure OMAX with Dataset $A$

\begin{tabular}{lcc}
\hline TOP-15\%STCOPs & Co-occurrence Rule & Conditional Probability \\
\hline$e_{1} e_{2}$ & $e_{2} \Rightarrow e_{1} e_{2}$ & 1.00 \\
& $e_{1} \Rightarrow e_{1} e_{2}$ & 0.83 \\
$e_{1} e_{6}$ & $e_{6} \Rightarrow e_{1} e_{6}$ & 0.93 \\
& $e_{1} \Rightarrow e_{1} e_{6}$ & 0.83 \\
$e_{3} e_{4}$ & $e_{4} \Rightarrow e_{3} e_{4}$ & 1.00 \\
& $e_{3} \Rightarrow e_{3} e_{4}$ & 0.83 \\
$e_{1} e_{3}$ & $e_{3} \Rightarrow e_{1} e_{3}$ & 1.00 \\
& $e_{1} \Rightarrow e_{1} e_{3}$ & 0.66 \\
$e_{2} e_{3}$ & $e_{3} \Rightarrow e_{2} e_{3}$ & 1.00 \\
& $e_{2} \Rightarrow e_{2} e_{3}$ & 0.63 \\
$e_{2} e_{6}$ & $e_{6} \Rightarrow e_{2} e_{6}$ & 0.75 \\
& $e_{2} \Rightarrow e_{2} e_{6}$ & 0.63 \\
$e_{3} e_{6}$ & $e_{3} \Rightarrow e_{3} e_{6}$ & 0.83 \\
& $e_{6} \Rightarrow e_{3} e_{6}$ & 0.62 \\
& $e_{4} \Rightarrow e_{3} e_{4} e_{6}$ & 1.00 \\
$e_{3} e_{4} e_{6}$ & $e_{3} e_{4} \Rightarrow e_{3} e_{4} e_{6}$ & 1.00 \\
& $e_{4} e_{6} \Rightarrow e_{3} e_{4} e_{6}$ & 1.00 \\
$e_{3} e_{6}$ & $e_{3} e_{6} \Rightarrow e_{3} e_{4} e_{6}$ & 0.97 \\
& $e_{3} \Rightarrow e_{3} e_{4} e_{6}$ & 0.83 \\
& $e_{4} \Rightarrow e_{4} e_{6}$ & 1.00 \\
$e_{1} e_{2} e_{3}$ & $e_{3} \Rightarrow e_{1} e_{2} e_{3}$ & 1.00 \\
& $e_{2} e_{3} \Rightarrow e_{1} e_{2} e_{3}$ & 0.88 \\
$e_{1} e_{2} e_{6}$ & $e_{1} e_{3} \Rightarrow e_{1} e_{2} e_{3}$ & 0.77 \\
$e_{1} e_{5}$ & $e_{2} \Rightarrow e_{1} e_{2} e_{3}$ & 0.63 \\
& $e_{6} \Rightarrow e_{1} e_{2} e_{6}$ & 0.62 \\
$e_{1} e_{3} e_{6}$ & $e_{2} e_{6} \Rightarrow e_{1} e_{2} e_{6}$ & 0.83 \\
& $e_{1} \Rightarrow e_{1} e_{5}$ & 0.77 \\
& $e_{3} e_{6} \Rightarrow e_{1} e_{3} e_{6}$ & 1.00 \\
& $e_{3} \Rightarrow e_{1} e_{3} e_{6}$ & 0.83 \\
$e_{1} e_{3} \Rightarrow e_{1} e_{3} e_{6}$ & 0.64 \\
$e_{6} \Rightarrow e_{1} e_{3} e_{6}$ & \\
& & \\
& & \\
& &
\end{tabular}

short-lasting. Similarly, the measure $O M A X$ also penalizes objects with smaller Intersection (common) volume. However, the measure OMAX does not penalize to the extent of the measure $J$. Thus, the measure $O M A X$ is more appropriate to datasets that contain event types with different life spans and areas (sizes).

\section{CONCLUSION AND FUTURE WORK}

In this article, we defined mining at most top-K\% spatiotemporal co-occurrence patterns for datasets with extended spatial representations that evolve over time. We analyzed measures $J$ and $O M A X$, which have the useful antimonotonic property, and we showed the effectiveness of these measures in filtering out pattern instances. We introduced our novel FastTop-K\%STCOPs algorithm and compared it with a naïve alternative, and showed the results highlighting the effectiveness of our pruning strategy to generate candidate patterns. We validated our algorithms using three real-life datasets, and showed the effectiveness of our algorithms in discovering top-K\% STCOPs (see Figures 5, 6, and 7). 
Although we got the inspiration for this work from Celik et al. [2006], there are several major differences between the TopMDCOP-Miner algorithm proposed in Celik et al. [2007] and our FastTop-K\%STCOPs for the following reasons: (1) The TopMDCOP-Miner algorithm works only with spatial-point datasets; in our work, event types are modeled as 3D spatial objects to capture different spatial and temporal characteristics of evolving extended spatial representations. (2) The TopMDCOP-Miner algorithm uses a time percentile threshold; we use a participation index percentile threshold to maintain the candidate list. (3) The TopMDCOP-Miner algorithm might not report all the top-K\% patterns, because they do not check the tie condition with percentile time prevalence threshold for the case when a newly generated size- $k+1$ pattern has a time prevalence value greater than the percentile time prevalence threshold, and there are size- $k$ patterns of the same time prevalence index as the newly generated size- $(k+1)$ pattern in the candidate list. The TopMDCOP-Miner algorithm will miss patterns for this case, while our FastTop-K\%STCOPs will report all the top-K\% patterns correctly (see Step 6 of our algorithm description). (4) The TopMDCOP-Miner algorithm does not discover the co-occurrence rules; our algorithms generate the co-occurrence rules as well.

For future work, we plan to investigate new computationally efficient algorithms for mining Top-K\%STCOPs for the Jaccard measure $(J)$. One such approach is to use an upper bound for $J$ and use a filter-and-refine strategy to discover a reduced set of pattern instances for the measure $J$ [Ganesan Pillai et al. 2013]. This additional pruning strategy will reduce the memory usage and execution time of the FastTopK\%STCOPs algorithm considerably over the current version.

\section{REFERENCES}

Rakesh Agrawal, Tomasz Imieliński, and Arun Swami. 1993. Mining association rules between sets of items in large databases. SIGMOD Record 22, 2, 207-216. DOI: http://dx.doi.org/10.1145/170036.170072

B. Aydin, D. J. Kempton, V. Akkineni, S. R. Gopovaram, and K. Ganesan Pillai, R. A. Angryk. 2014. Spatiotemporal indexing techniques for efficiently mining spatiotemporal co-occurrence patterns. In Proceedings of the 2nd IEEE International Conference on Big Data (ICBD-IEEE'14), Workshop on Workshop on Management, Search and Mining of Massive Repositories of Solar Astronomy Data (SABID). 1-10.

Mete Celik, Shashi Shekhar, James P. Rogers, James A. Shine, and James M. Kang. 2007. Mining at most top-k mixed-drove spatio-temporal co-occurrence patterns: A summary of results. In Proceedings of the Workshop on Spatio-Temporal Data Mining (ICDE'07).

Mete Celik, Shashi Shekhar, James P. Rogers, James A. Shine, and Jin Soung Yoo. 2006. Mixed-drove spatio-temporal co-occurrence pattern mining: A summary of results. In Proceedings of the 6th International Conference on Data Mining (ICDM'06). IEEE Computer Society, Washington, DC, 119-128. DOI : http://dx.doi.org/10.1109/ICDM.2006.112

Yin Cheung and Ada Fu. 2004. Mining frequent itemsets without support threshold: With and without item constraints. In IEEE Transactions on Knowledge and Data Engineering 16, 1069-2004.

L. Egghe and C. Michel. 2002. Strong similarity measures for ordered sets of documents in information retrieval. Information Processing and Management 38, 6, 823-848. DOI:http://dx.doi.org/10.1016/ S0306-4573(01)00051-6

Ada Fu, Renfrew Wang wai Kwong, Fu Renfrew, Wang wai Kwong, and Jian Tang. 2000. Mining N-most interesting itemsets. In International Symposium on Methodologies for Intelligent Systems.

Karthik Ganesan Pillai. 2014a. https://www.cs.montana.edu/ k.ganesanpillai/

Karthik Ganesan Pillai. 2014b. Mining spatiotemporal co-occurrence patterns from massive data sets with evolving regions. PhD dissertation. Montana State University.

Karthik Ganesan Pillai, Rafal A. Angryk, and Berkay Aydin. 2013. A filter-and-refine approach to mine spatiotemporal co-occurrences. In Proceedings of the 21st ACM SIGSPATIAL International Conference on Advances in Geographic Information Systems (ACM SIGSPATIAL GIS'13). 114-123.

Karthik Ganesan Pillai, Rafal A. Angryk, Juan M. Banda, Michael A. Schuh, and Tim Wylie. 2012. Spatiotemporal co-occurrence pattern mining in data sets with evolving regions. In ICDM Workshops. 805-812.

Karthik Ganesan Pillai, Rafal A. Angryk, Juan M. Banda, Tim Wylie, and Michael A. Schuh. 2014. Spatiotemporal co-occurrence rules. In New Trends in Databases and Information Systems. 
Advances in Intelligent Systems and Computing, Vol. 241. Springer International Publishing, 27-35. DOI : http://dx.doi.org/10.1007/978-3-319-01863-8_3

HEK. Nov. 2013. Heliophysics events registry. Retrieved August 19, 2016 from http://www.lmsal.com/ isolsearch.

Yu Hirate, Eigo Iwahashi, and Hayato Yamana. 2004. TF 2 P-growth: An efficient algorithm for mining frequent patterns without any thresholds. In Proceedings of Workshop on Alternative Techniques for Data Mining and Knowledge Discovery.

R. F. Howard, J. W. Harvey, and S. Forgach. 1990. Solar surface velocity fields determined from small magnetic features. Solar Physics 130, 1-2, 295-311.

Yan Huang, Shashi Shekhar, and Hui Xiong. 2004. Discovering colocation patterns from spatial data sets: A general approach. IEEE Transactions on Knowledge and Data Engineering 16, 12, 1472-1485.

N. Hurlburt, M. Cheung, C. Schrijver, L. Chang, S. Freeland, S. Green, C. Heck, A. Jaffey, A. Kobashi, D. Schiff, J. Serafin, R. Seguin, G. Slater, A. Somani, and R. Timmons. 2010. Heliophysics event knowledgebase for the Solar Dynamics Observatory (SDO) and beyond. Solar Physics 275, 1, 67-78. DOI : http://dx.doi.org/10.1007/s11207-010-9624-2

D. J. Kempton, K. Ganesan Pillai, and R. A. Angryk. 2014. Iterative refinement of multiple targets tracking of solar events. In Proceedings of the 2nd IEEE International Conference on Big Data (ICBD-IEEE'14), Workshop on Workshop on Management, Search and Mining of Massive Repositories of Solar Astronomy Data (SABID). 36-44.

V. Lakshmanan, J. Zhang, and K. Howard. 2010. A Technique to Censor Biological Echoes in Radar Reflectivity Data. Vol. 49. 435-462.

R. Lamb, Rafal Angryk, and P. C. H. Martens. 2008. An example-based image retrieval system for the TRACE repository. In Proceedings of the 19th International Conference on Pattern Recognition (ICPR'08), 1-4.

Stephanie Langhof and Tore Straume. 2011. Workshop report on space weather risks and society. In Report of a Workshop Sponsored by and held at NASA Ames Research Center.

Christopher D. Manning and Hinrich Schütze. 1999. Foundations of Statistical Natural Language Processing. MIT Press, Cambridge, MA.

P. C. H. Martens, G. D. R. Attrill, A. R. Davey, A. Engell, S. Farid, P. C. Grigis, J. Kasper, K. Korreck, S. H. Saar, A. Savcheva, Y. Su, P. Testa, M. Wills-Davey, P. N. Bernasconi, N.-E. Raouafi, V. A. Delouille, J. F. Hochedez, J. W. Cirtain, C. E. DeForest, R. A. Angryk, I. De Moortel, T. Wiegelmann, M. K. Georgoulis, R. T. J. McAteer, and R. P. Timmons. 2012. Computer Vision for the Solar Dynamics Observatory (SDO). Solar Physics 275, 1, 79-113.

Chung Ngan, Tsang Lam, Wing Wong, Raymond Chi, Chee Fu, and Ada Wai. 2005. Mining N most interesting itemsets without support threshold by the COFI tree. International Journal of Business Intelligence and Data Mining 1, 1, 88-106. DOI : http://dx.doi.org/10.1504/IJBIDM.2005.007320

M. A. Schuh, R. A. Angryk, K. Ganesan Pillai, J. M. Banda, and P. C. H. Martens. 2013. A large-scale solar image dataset with labeled event regions. In International Conference on Image Processing (ICIP'13). 4349-4353.

Shashi Shekhar and Sanjay Chawla. 2002. Spatial Databases: A Tour. Pearson Education, New York, NY.

Shashi Shekhar and Yan Huang. 2001. Discovering spatial co-location patterns: A summary of results. In Lecture Notes in Computer Science, Vol. 2121. Berlin. 236-256.

Pang-Ning Tan, Michael Steinbach, and Vipin Kumar. 2005. Introduction to Data Mining. Addison-Wesley Longman Publishing Co., Inc., Boston, MA.

P. J. Taylor. 1977. Quantitative Methods in Geography: An Introduction to Spatial Analysis. Houghton Mifflin, Boston, MA.

Jin Soung Yoo and Mark Bow. 2009. Finding N-most prevalent colocated event sets. In Proceedings of the 11th International Conference on Data Warehousing and Knowledge Discovery (DaWaK'09). Springer-Verlag, Berlin, 415-427. DOI : http://dx.doi.org/10.1007/978-3-642-03730-6_33

Received November 2013; revised December 2015; accepted May 2016 